\title{
Higher-Order Statistics for the Detection of Small Objects in a Noisy Background Application on Sonar Imaging
}

\author{
F. Maussang, ${ }^{1}$ J. Chanussot, ${ }^{2}$ A. Hétet, ${ }^{3}$ and M. Amate ${ }^{3}$ \\ ${ }^{1}$ Groupe d'Electromagnétisme Appliqué (GEA), PST Ville d'Avray, Université Paris X-Nanterre, 1 chemin Desvallières, \\ 92410 Ville d'Avray, France \\ ${ }^{2}$ Laboratoire des images et des signaux (LIS) GIPSA, École Nationale Supérieure d'Ingénieurs Électriciens de Grenoble, \\ Institut National Polytechnique de Grenoble (INPG), Domaine Universitaire, BP 46, 38402 Saint-Martin-d'Hères Cedex, France \\ ${ }^{3}$ Groupe d'Etudes Sous-Marines de l'Atlantique, DGA/DET/GESMA, BP 42, 29240 Brest Armées, France
}

Received 21 June 2006; Revised 10 November 2006; Accepted 21 November 2006

Recommended by Christoph Mecklenbräuker

\begin{abstract}
An original algorithm for the detection of small objects in a noisy background is proposed. Its application to underwater objects detection by sonar imaging is addressed. This new method is based on the use of higher-order statistics (HOS) that are locally estimated on the images. The proposed algorithm is divided into two steps. In a first step, HOS (skewness and kurtosis) are estimated locally using a square sliding computation window. Small deterministic objects have different statistical properties from the background they are thus highlighted. The influence of the signal-to-noise ratio (SNR) on the results is studied in the case of Gaussian noise. Mathematical expressions of the estimators and of the expected performances are derived and are experimentally confirmed. In a second step, the results are focused by a matched filter using a theoretical model. This enables the precise localization of the regions of interest. The proposed method generalizes to other statistical distributions and we derive the theoretical expressions of the HOS estimators in the case of a Weibull distribution (both when only noise is present or when a small deterministic object is present within the filtering window). This enables the application of the proposed technique to the processing of synthetic aperture sonar data containing underwater mines whose echoes have to be detected and located. Results on real data sets are presented and quantitatively evaluated using receiver operating characteristic (ROC) curves.
\end{abstract}

Copyright (c) 2007 F. Maussang et al. This is an open access article distributed under the Creative Commons Attribution License, which permits unrestricted use, distribution, and reproduction in any medium, provided the original work is properly cited.

\section{INTRODUCTION}

Higher-order statistics (HOS) are largely used in signal processing and have already been applied to various domains: astronomy (that provided pioneering applications), but also seismic data processing, communication and, more recently, geophysics, speech, radar, and sonar signal processing and analysis. In the last decade, several journals have published special issues on these emerging techniques [1-3]. As a matter of fact, HOS allow the solving of problems that first- and second-order statistics fail to solve. For example, they enable linear system identification by blind deconvolution $[4,5]$, and nonlinear identification (Volterra filter) [6]. They are used in nonstationary signals analysis [7], array processing [8], and source separation [9-11].

A much scarcer literature addresses the use of HOS in the field of image processing (considering images as bidimensional signals). Jacovitti [12] presented applications of
HOS to image decomposition, blind deconvolution, coding and pattern recognition. Some studies were also made on texture analysis $[13,14]$ and segmentation by data clustering [15]. Carrato and Ramponi [16] presented a Skewness-ofGaussian edge extractor applied on images. This method uses the crossing of a skewness operator by zero (corresponding to a symmetric distribution) to detect edges in images with good performances and robustness [17]. The most interesting paper regarding our application is proposed by Alexandrou et al. [18]: a coefficient of excess, the kurtosis (4thorder statistical value), is studied in order to model the nonGaussian reverberation hypothesis. But, as raised by the authors, it is difficult to differentiate this excess, induced by an inaccurate modeling of the background with a Gaussian law, from a potential coherent component embedded in the reverberation. Moreover, this difficulty increases as the number of samples decreases, which does not allow a local estimation in order to detect small coherent elements (echoes). 
In this paper, ${ }^{1}$ a new statistical detection method on images is proposed. It aims at detecting small targets, modeled as deterministic regions, in a noisy background which is previously statistically modeled by a Weibull law. It is based on higher-order statistical properties of the image. The global detection process can be divided into four main steps:

(1) HOS (skewness or kurtosis) are locally estimated on a square computation window sliding all over the image;

(2) the results are focused with a matched filter in order to accurately locate the sought regions;

(3) a rebuilding of the sought regions is performed using a morphological dilation;

(4) finally, a gray-level threshold is applied to detect the objects (ROC curves).

The paper is organized as follows. In Section 2, some properties of the used HOS are recalled and two classical estimators (for the skewness and the kurtosis, resp.) are defined and presented. In Section 3, the use of HOS for the purpose of detection is discussed in function of the signal-to-noise ratio (SNR). In Section 4, the focusing process used in order to obtain an accurate localization of the different regions of interest is presented. In the last section, the proposed method is tested on real sonar data containing various underwater objects, both lying on the sea-bed and buried, after a presentation of the statistical specificities of these images. In particular, this requires the derivation of the theoretical HOS estimators in the case of a Weibull distribution.

\section{HOS ESTIMATORS}

\subsection{Definitions}

The two most classically used HOS are the skewness (derived from the 3rd-order moment) and the kurtosis (derived from the 4th-order moment) [19]. One should underline that beyond these two standard statistics, other statistics with an order greater than 4 can be mathematically defined. However, these statistics are extremely difficult to estimate in a reliable and robust way and are thus practically never used. Noting $\mu_{X(r)}$ as the $r$ th order central moment of a random variable $X$, the definition of the skewness is given by

$$
S_{X}=\frac{\mu_{X(3)}}{\mu_{X(2)}^{3 / 2}}
$$

A definition of the kurtosis is given by

$$
K_{X}=\frac{\mu_{X(4)}}{\mu_{X(2)}^{2}}-3
$$

The skewness measures the symmetry of a random distribution, while the kurtosis measures whether the data distribution is peaked or flat relative to a normal distribution. These statistics are theoretically null for the normal distribution.

\footnotetext{
${ }^{1}$ This paper is on results obtained by F. Maussang during his Ph.D. in LIS.
}

\subsection{Estimators}

To estimate the skewness and the kurtosis on a sample $X$ of finite size $N, k$-statistics $k_{X(r)}$ can be used. $k_{r}$ is defined as the unique symmetric unbiased estimator of the cumulant $\kappa_{X(r)}$ on $X$ [19]. An unbiased estimator of the skewness is then given by

$$
\widehat{S}_{X}=\frac{k_{X(3)}}{k_{X(2)}^{3 / 2}}
$$

Defining the $r$ th sample central moment of $X$ by the following expression:

$$
m_{X(r)}=\frac{1}{N} \sum_{i=1}^{N}\left(x_{i}-\bar{x}\right)^{r}
$$

where $\bar{x}=(1 / N) \sum_{i=1}^{N} x_{i}$ and $x_{i}$ are the $N$ samples of $X$, we can derive another definition of this estimator. Actually, considering the relationships between $k_{X(r)}$ and $m_{X(r)}$, we have

$$
\widehat{S}_{X}=\frac{\sqrt{N(N-1)}}{N-2} \frac{m_{X(3)}}{m_{X(2)}^{3 / 2}} \text {. }
$$

In the same way, we derive the following estimator for the kurtosis:

$$
\hat{K}_{X}=\frac{k_{X(4)}}{k_{X(2)}^{2}}=\frac{(N+1)(N-1)}{(N-2)(N-3)} \frac{m_{X(4)}}{m_{X(2)}^{2}}-\frac{3(N-1)^{2}}{(N-2)(N-3)} .
$$

Asymptotic statistical properties are studied for high values of $N$. Firstly, we can mention that these estimators are biased in the first order and that they are correlated (the bias being dependent on higher-order moments). However, exact results can be derived in the Gaussian case. In this case, $M$ and $V$ being, respectively, the mean and the variance, we have

$$
\begin{gathered}
M\left(\hat{S}_{X}\right)=0, \\
M\left(\hat{K}_{X}\right)=0, \\
V\left(\hat{S}_{X}\right)=\frac{6 N(N-1)}{(N-2)(N+1)(N+3)} \approx \frac{6}{N}, \\
V\left(\hat{K}_{X}\right)=\frac{24 N(N-1)^{2}}{(N-3)(N-2)(N+3)(N+5)} \approx \frac{24}{N} .
\end{gathered}
$$

In the general case, there is no analytical expression for unbiased estimators independently from the probability density function of the random value. However, one should note that in the case of a normal distribution, the estimators are unbiased. Nevertheless, variances of these estimators are relatively high and it is well known that a reliable estimation requires a large set of samples.

\section{HOS FOR DETECTION}

To illustrate the detection method proposed in this paper, it is first tested on a simple synthetic image $(128 \times 128$ pixels $)$. 
It consists of a square of size $11 \times 11$, with a constant amplitude $A$, surrounded by a noisy background. The noise in this image has a central Gaussian distribution with a variance $\sigma_{B}^{2}$. Its probability density function is given by

$$
G_{B}(B)=\frac{1}{\sigma_{B} \sqrt{2 \pi}} \exp \left(-\frac{B^{2}}{2 \sigma_{B}^{2}}\right)
$$

and its $r$ th noncentral moments $\mu_{B(r)}^{\prime}$ are

$$
\mu_{B(r)}^{\prime}= \begin{cases}\sigma_{B}^{r} \frac{r !}{(r / 2) ! 2^{r / 2}} & \text { if } r \text { is even } \\ 0 & \text { otherwise. }\end{cases}
$$

Note that combining these noncentral moments leads to the nullity of the skewness and kurtosis for a normal distribution.

Regarding the deterministic square of amplitude $A$, its $r$ th noncentral moments $\mu_{D(r)}^{\prime}$ are

$$
\mu_{D(r)}^{\prime}=A^{r}
$$

Considering the deterministic square as a signal surrounded by noise, the SNR can be defined as

$$
\rho=\frac{A}{\sigma_{B}}
$$

and in decibels:

$$
\rho_{d B}=10 \log _{10}\left(\frac{A^{2}}{\sigma_{B}^{2}}\right)=20 \log _{10}(\rho) .
$$

Skewness and kurtosis are invariant by a scale shift. This is of the utmost importance: since, in the following, the HOS are estimated locally, the proposed method is invariant to varying offset in the image. Consequently, if the noise is modeled by a noncentral Gaussian of mean $\mu$ and variance $\sigma^{2}$, described by the following density probability function:

$$
G_{B}^{\prime}(B)=\frac{1}{\sigma_{B} \sqrt{2 \pi}} \exp \left\{-\frac{(B-\mu)^{2}}{2 \sigma_{B}^{2}}\right\}
$$

all the results obtained in the following remain valid with an SNR defined as

$$
\rho^{\prime}=\frac{|A-\mu|}{\sigma_{B}} .
$$

\subsection{Local properties of the HOS}

Skewness and kurtosis estimators previously described in (5) and (6) are used in this paper to detect small deterministic regions in a noisy image. For this purpose, these HOS are locally estimated for each pixel using a square sliding window, composed of $N$ pixels, the current pixel being in the center.

To model the situation and explain the results obtained when applied on the images, $p \in[0,1]$ is defined as the proportion of deterministic pixels in the computation window (Figure 1). Consequently, $(1-p)$ is the proportion of random

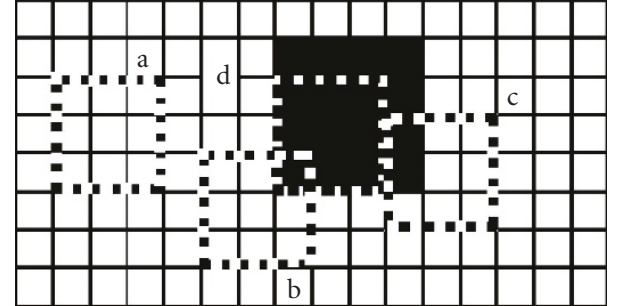

Figure 1: Various values of parameter $p$ : (a) $p=0$, (b) $p=1 / 9$, (c) $p=2 / 9$, (d) $p=1$ (black pixels $=$ deterministic region, white pixels = background).

values (pixels belonging to the noisy background). In the following, the local statistical properties (moments) of the deterministic region and the noisy background in the computation window are assumed to be the same as the global ones presented in the previous section. This is achieved when the size of the computation window is large enough. In particular, this size should be greater than the maximum size of the sought deterministic regions. Note that in practical cases, the value of $p$ for one given position of course remains unknown.

Considering $\mu_{D(r)}^{\prime}, \mu_{B(r)}^{\prime}$, and $\mu_{W(r)}^{\prime}$ the $r$ th noncentral moments computed on the "deterministic-part" of the filtering window, on the "noisy background part," and on the whole window, respectively, the following relation holds:

$$
\mu_{W(r)}^{\prime}=p \cdot \mu_{D(r)}^{\prime}+(1-p) \cdot \mu_{B(r)}^{\prime} .
$$

Therefore, we can combine the definitions of the skewness and kurtosis in (1) and (2), the noncentral moments of the different parts of the window in (9) and (10), the definition of the SNR $\rho$ (11), and the relationships between the central and noncentral moments [19]. Using the previous model, we derive the following expressions for the skewness $S_{W}$ and the kurtosis $K_{W}$ estimated on the computation window:

$$
\begin{gathered}
S_{W}(\rho, p)=\frac{p}{\sqrt{1-p}} \frac{(1-2 p) \rho^{3}-3 \rho}{\left(p \rho^{2}+1\right)^{3 / 2}}, \\
K_{W}(\rho, p)=\frac{p}{1-p} \frac{\left(1-6 p+6 p^{2}\right) \rho^{4}-6(1-2 p) \rho^{2}+3}{\left(p \rho^{2}+1\right)^{2}} .
\end{gathered}
$$

The evolution of these HOS in function of $p$ and the $\operatorname{SNR} \rho$ is plotted on Figure 2. Different behaviors are observed depending on whether the SNR is high or low. The skewness has low values for low SNRs, but, for high SNRs, it has high values for low values of $p$ and negative values for high values of $p$ (close to 1). This is confirmed by Figure 3(a) where we can observe the skewness getting close to zero for low SNRs (below $0 \mathrm{~dB}$ for low $p$ and below $-20 \mathrm{~dB}$ for high $p$ ) and taking higher values for high SNRs (above $20 \mathrm{~dB}$ ). Intermediate values are obtained for intermediate values of the SNR. For the kurtosis (Figure 3(b)), opposed behaviors are observed depending on whether the SNR is low or high, with an intermediate area between 0 and $20 \mathrm{~dB}$. Considering these properties, the detection problem is addressed in the next three 


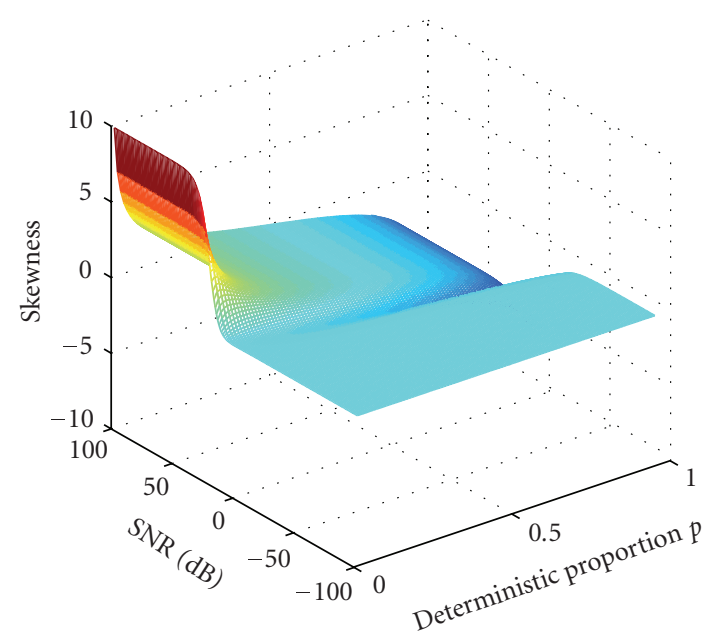

(a) Skewness

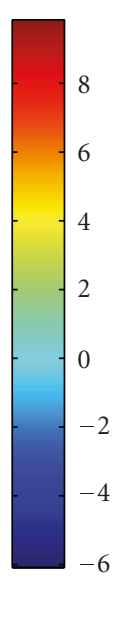

FIGURE 2: HOS in function of the deterministic proportion $p$ and the SNR.

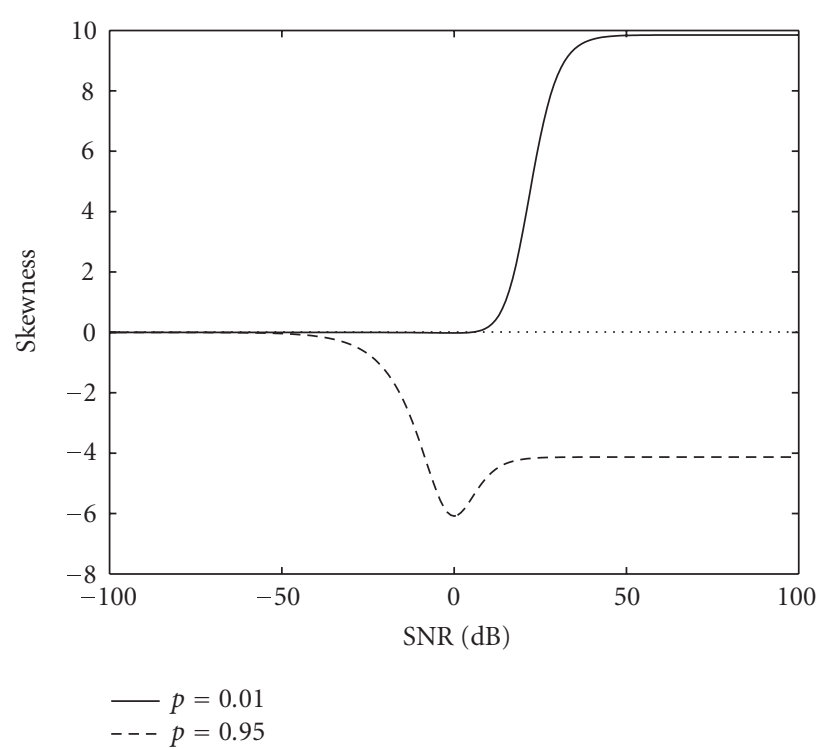

(a) Skewness

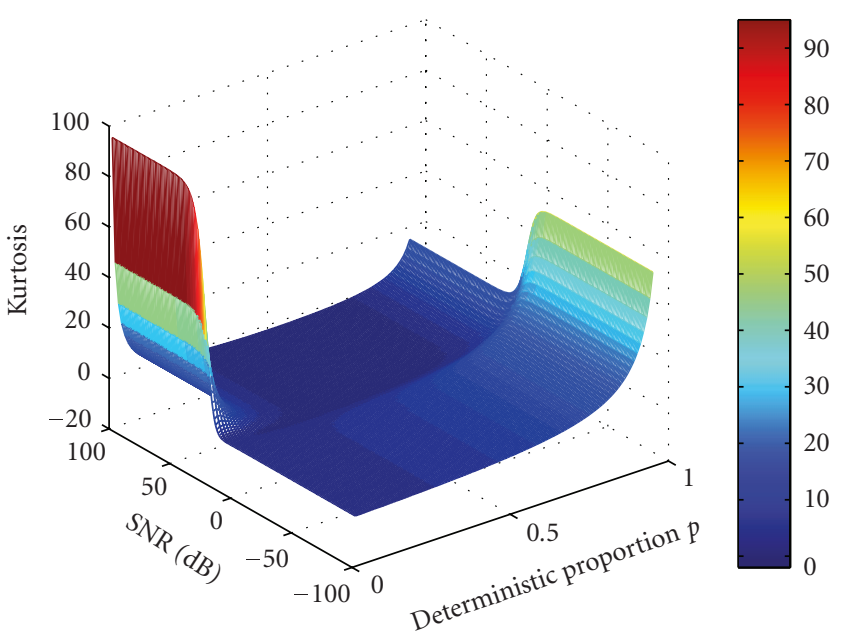

(b) Kurtosis (1) (1) 


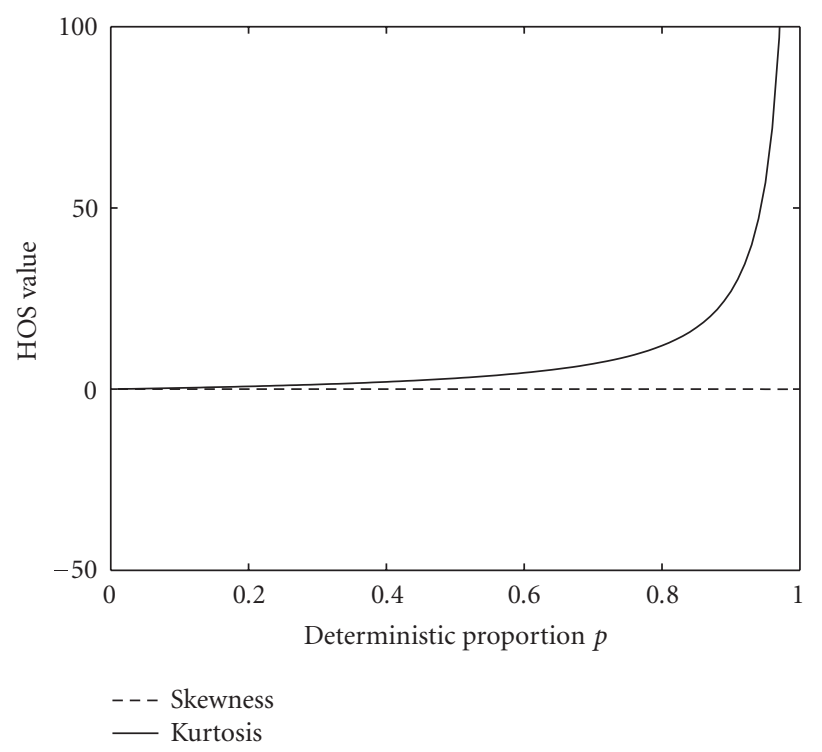

FIgure 4: Skewness (dashed line) and kurtosis (solid line) in function of parameter $p$ for SNR $=-60 \mathrm{~dB}$.

when $p$ goes from zero to one, and the skewness values are close to zero. This result is illustrated on Figure 5, representing an example of probability density function obtained on a window with a low SNR, showing a symmetric density, hence a low skewness, with a peak, hence a high kurtosis.

These approximations also explain the results obtained with the synthetic image (Figure 6). Note that on this example only used for illustration, the detection would be trivial since the "target" is clearly visible. However, we must underline that in real cases the regions of interest can be composed of a very few pixels in a very large image, thus with an extremely low visibility.

The skewness image (Figure 6(b)) only consists of low values, without any clearly contrasted region emerging from the noisy background. On the contrary, the kurtosis has low values in the regions corresponding to the noisy background and higher values around the center of the deterministic region. This is consistent with the predicted increase of the kurtosis with $p$. Actually, the closer is the filtering window to the center of the object, the higher is the deterministic proportion $p$ in the window. Note that the previous approximations remain valid when no deterministic region is present (e.g., in the noisy background, with $p=0$ ). Therefore, in the case of low SNRs, a detection of small deterministic objects can be made by performing a simple threshold selecting the high values of the kurtosis. On the other hand, skewness does not lead to any satisfactory detection.

Given the influence of $p$ on the kurtosis, the smaller is the computation window, the higher are the values of the kurtosis, and the better is the detection. This is illustrated on Figure 7 where we can see higher and more centered values of the kurtosis with a $13 \times 13$ window than with a $41 \times 41$ one. But, as stated in Section 2.2 (7), the smaller is this window, the higher is the variance of the estimator, and the less robust

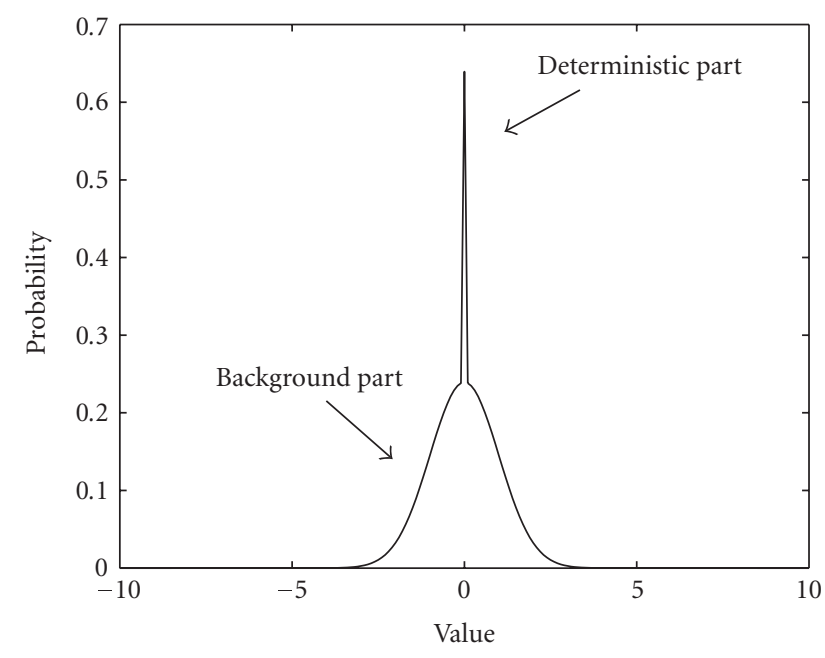

FIgure 5: Example of probability density function on a computation window for a low SNR $(p=0.4)$.

is the detection. This is illustrated on Figure $7(a)$ where the maximum is about 8.2 when the theoretical result predicts $p_{\max }=11 \times 11 / 13 \times 13=121 / 169$ which leads to a maximum value of $3 p_{\text {max }} /\left(1-p_{\text {max }}\right) \approx 7.56$ for the kurtosis.

To illustrate these comments, two numerical values are computed on the results. The first one is the variance of the HOS on the background (to the exclusion of all the pixels where the filtering window actually meets the target). The second criterion measures the contrast: it is the ratio between the maximum value of the HOS on the image (absolute value) and the standard deviation of the background (square root of the variance previously estimated). This parameter allows to estimate the quality of the detection, bound with the enhancement of the regions of interest compared with the background. Table 1 presents the results obtained on the HOS images with an SNR of $-60 \mathrm{~dB}$ and different sizes of computation window.

As previously mentioned, the narrower is the computation window (and the lower is the number of samples), the higher are the variances of the HOS estimators. This induces an increase of the variance of the background. This is even higher for the kurtosis, as predicted by (7) (with a ratio close to 4 , especially for large windows). However all the variances are significantly lower than the variance of the original image (close to 1, the Gaussian noise being of standard deviation 1). Furthermore, the contrast values confirm our previous visual observations regarding the kurtosis: the detection is easier for small computation windows. Regarding the skewness, the contrast has lower values, whatever the size of the window, confirming its low potential in terms of detection.

To conclude with the detection in the case of a low SNRs, the skewness is of little interest, its values always being close to zero, whether there is an object or not. On the contrary, the kurtosis gives interesting results for the detection. However, a trade-off has to be found for the size of the computation window. A large size ensures a low variance of estimation, but gives low values of kurtosis. A small size gives higher values of 


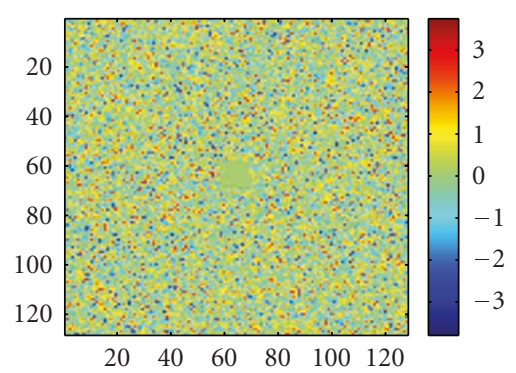

(a) Synthetic image

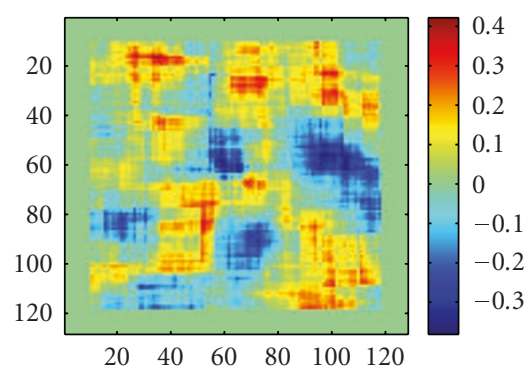

(b) Skewness

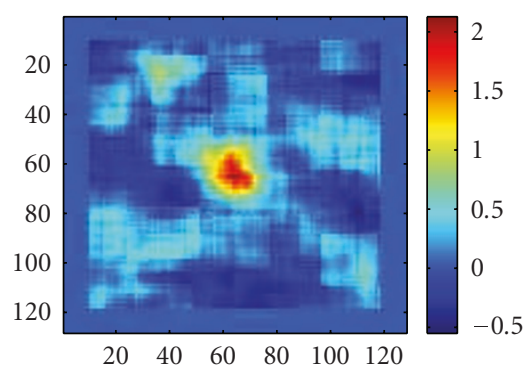

(c) Kurtosis

Figure 6: HOS evaluated on $21 \times 21$ windows $(S N R=-60 \mathrm{~dB})$.

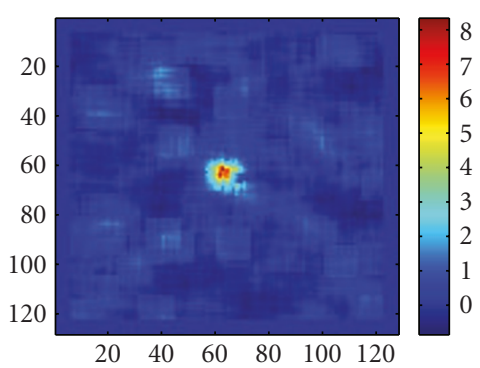

(a) $13 \times 13$

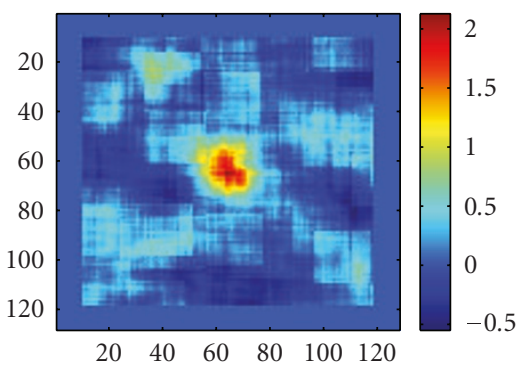

(b) $21 \times 21$

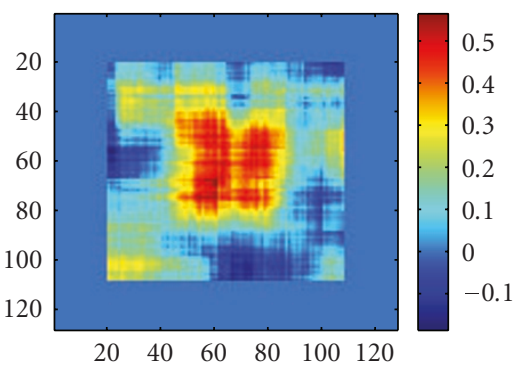

(c) $41 \times 41$

FIGURE 7: Kurtosis images obtained with different sizes of window (SNR $=-60 \mathrm{~dB})$.

TABLE 1: Detection performances of the HOS (variance of the background and contrast), with an SNR of $-60 \mathrm{~dB}$, in function of the size of the computation window.

\begin{tabular}{c|cc|cc}
\hline \multirow{2}{*}{ Size of the window } & \multicolumn{2}{|c|}{ Skewness } & \multicolumn{2}{c}{ Kurtosis } \\
& Variance & Contrast & Variance & Contrast \\
\hline $13 \times 13$ & 0.0282 & 7.94 & 0.115 & 24.6 \\
$21 \times 21$ & 0.0099 & 4.23 & 0.0375 & 11.0 \\
$41 \times 41$ & 0.0011 & 5.42 & 0.0039 & 8.99 \\
\hline
\end{tabular}

kurtosis, and thus a better detection, but with a high variance of estimation.

\subsection{Application to the detection of small objects: the case of high SNRs}

In this section, we consider images containing small objects with a high SNR. As suggested by the curves on Figure 3, SNRs greater than $40 \mathrm{~dB}$ are considered as high SNRs. For an illustrative purpose, HOS are estimated in a synthetic image with a $50 \mathrm{~dB}$ SNR (Figure 9(a)).

For high SNRs $(\rho \rightarrow \infty)$, we derive the following approximate expressions of the previously presented equations (16):

$$
\begin{gathered}
S_{W}(p) \approx \lim _{\rho \rightarrow+\infty} S_{W}(\rho, p)=\frac{1-2 p}{\sqrt{p(1-p)}}, \\
K_{W}(p) \approx \lim _{\rho \rightarrow+\infty} K_{W}(\rho, p)=\frac{1-6 p+6 p^{2}}{p(1-p)} .
\end{gathered}
$$

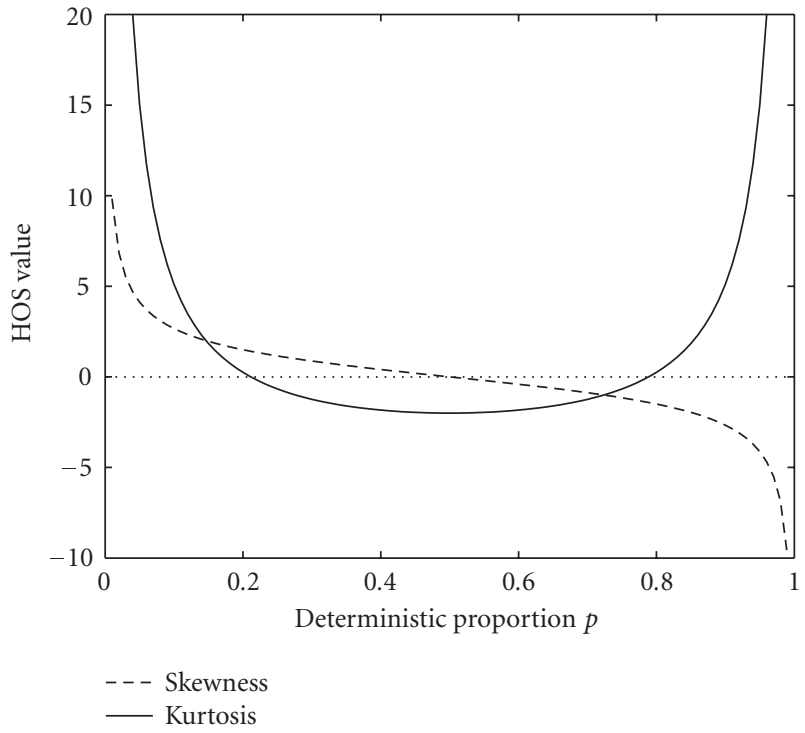

Figure 8: Skewness (dashed line) and kurtosis (solid line) for $\mathrm{SNR}=50 \mathrm{~dB}$

This is illustrated on Figure 8. For an SNR of $50 \mathrm{~dB}$ : the kurtosis decreases from infinity to -2 when $p$ increases from 0 to 0.5 . It again increases to infinity when $p$ increases from 0.5 to 1 . The skewness has a different behavior: it decreases 


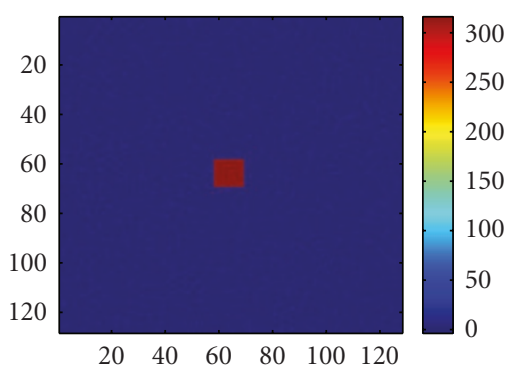

(a) Synthetic image

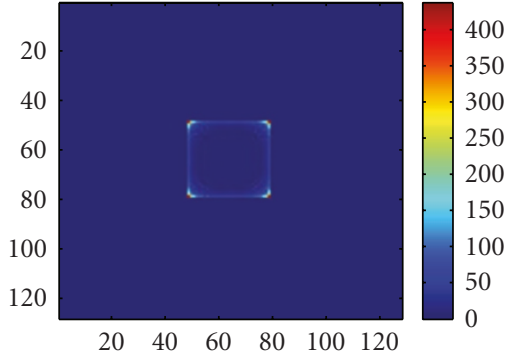

(b) Skewness

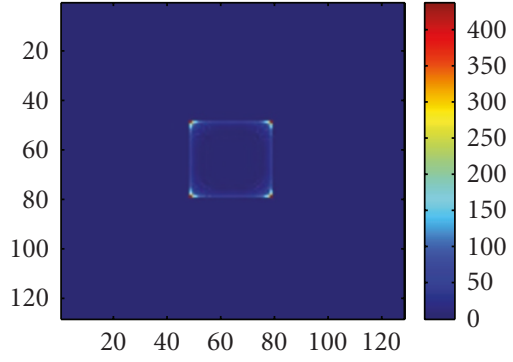

(c) Kurtosis

FIgURE 9: HOS evaluated on $21 \times 21$ windows $(\mathrm{SNR}=50 \mathrm{~dB})$.

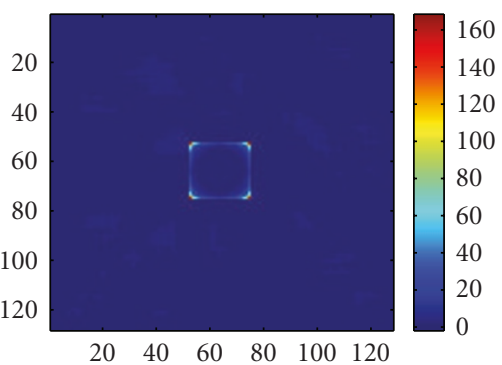

(a) $13 \times 13$

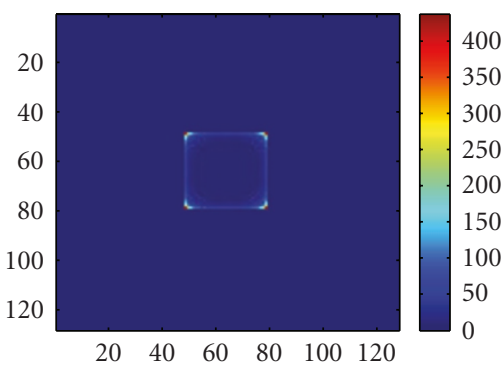

(b) $21 \times 21$

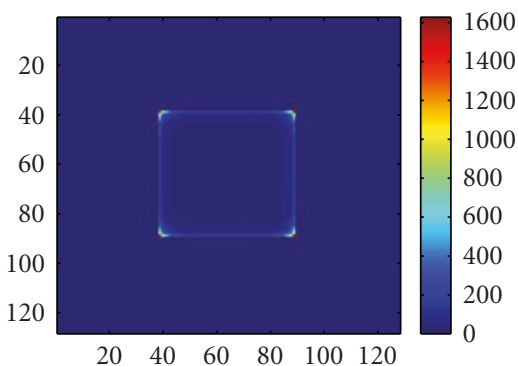

(c) $41 \times 41$

Figure 10: Kurtosis images obtained with different sizes of window ( $\mathrm{SNR}=50 \mathrm{~dB})$.

from plus to minus infinity as $p$ goes from 0 to 1 , with a zero crossing for $p=0.5$.

Figure 9 illustrates this situation and presents the results obtained for the skewness and the kurtosis (Figures 9(b) and $9(\mathrm{c})$, resp.) in the case of a synthetic test image with a high SNR (Figure 9(a)). We can make the following observations.

(i) In a noisy background, the HOS have small values. This corresponds to the nullity of the HOS for the Gaussian distribution (note that in this specific case, the approximations proposed in (18) and (19) do not hold anymore).

(ii) Square structures are observed around the object of interest. They are composed of pixels with high values, the highest being in the corners.

The size of these squares corresponds to the size of the deterministic region plus the size of the computation window. Considering the deterministic region as a square of side $n_{D}$ and a square computation window of side $n_{W}$ (the total number of pixels inside the computation window being $N=n_{D}^{2}$ ), the side $n_{S}$ of the square appearing in the HOS images is given by

$$
n_{S}=n_{D}+n_{W}-1
$$

For example, on Figure 9, the object is of size $11 \times 11$. This leads to a $31 \times 31$ frame.

As seen on Figure 8, the estimators reach their maximal values for the minimal values of parameter $p$. This corresponds to the case where one single pixel of the object of interest is included in the computation window (i.e., the corner of the structure, when the computation window starts overlapping it). When the number of deterministic pixels included inside the filtering window increases (i.e., when the window keeps on sliding towards the center of the object), the value of the estimators decreases. That explains the shape of the square structures observed in the result images and the decreasing values along the edges and inside the square. From (18) and (19), for low values of $p$, skewness can be approximated by $1 / \sqrt{p}$ and kurtosis by $1 / p$. This explains that for a $21 \times 21$ window, the highest values in the skewness image is close to 21 (corresponding to $p=1 /(21 \times 21)$ ) and 441 for the kurtosis. As a consequence, the higher is the size of the computation window, the higher is the value of the maximum on the skewness and the kurtosis image. Figure 10 illustrates the effect of the size of the computation window on the kurtosis estimation results: the larger is the window, the larger is the resulting square and the higher is the maximum value (check the scales). When $p$ goes above 0.5 (the computation window contains more pixels belonging to the deterministic object than to the noisy background), the kurtosis starts to increase again, which explains increasing values near the center of the sought region on Figure 10(a). To avoid this case, we use a computation window whose side is twice as large as the largest possible dimension of the sought objects. Assuming we are only interested in detecting small objects, this is easily fulfilled. Furthermore, as described in Section 2.2, the use of a too small filtering window does not lead to a robust estimation. However, this size should not be too large either. Actually, as previously observed, the size of the square structure on the HOS image is equal to the 


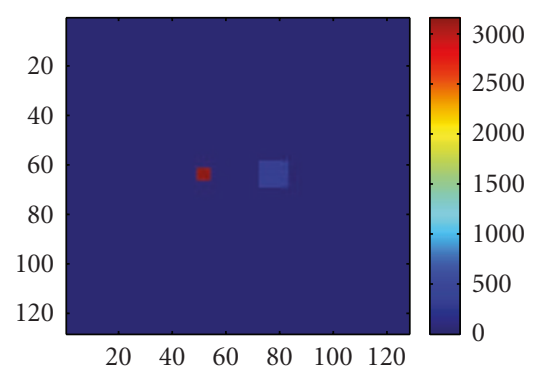

(a) Synthetic image

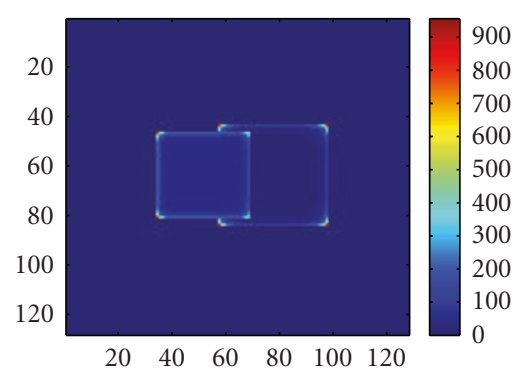

(b) $31 \times 31$

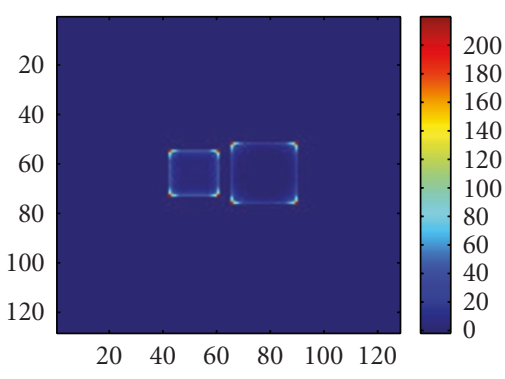

(c) $15 \times 15$

FIGURE 11: Kurtosis images obtained with different sizes of window on a synthetic image with two regions (left object: $\mathrm{SNR}=70 \mathrm{~dB}$, right object: SNR $=50 \mathrm{~dB})$.

size of the deterministic region plus the size of the computation window. If one given image contains several objects of interest, the corresponding structures in the HOS images may overlap if the filtering window is too large. This situation is illustrated on Figure 11 with two regions of different amplitudes and different sizes $(5 \times 5$ on the left, $11 \times 11$ on the right), with a 21 pixel wide gap in between. Note on Figure 11(b) the independence of the kurtosis value from the SNR (both objects lead to the same maximum value).

The contrasts reported on Table 2 confirm these observations, the contrast increasing with the size of the computation window. The variances being estimated on the noisy background, the variances reported on Table 1 do not depend on the SNR. The contrast estimated on the original image corresponds to the SNR (about 316.2 for $50 \mathrm{~dB}$ ). The contrast obtained with the HOS is largely greater, especially for a large window. This highlights the interest of a detection using these statistical values. Finally, note that, for a given size of window, the contrast obtained with the kurtosis is greater than with the skewness. This is also confirmed by the derived approximations that give higher values of the kurtosis for low values of $p$ (lower than approximately $0.15,(2-\sqrt{2}) / 4$ exactly).

As a conclusion to this section, both the skewness and the kurtosis allow the detection of deterministic regions. However, the maximum values being in the corners of a square located around the region of interest, the precise localization of the sought regions requires some post processing. This "focusing" of the resulting frames to the real position of the objects will be studied in Section 4. Moreover, the choice of the size of the computation window appears as a trade-off between the size of the deterministic regions that must be detected and the space separating two different objects.

\subsection{Application to the detection of small objects: the case of intermediate SNRs}

SNRs between $-20 \mathrm{~dB}$ and $40 \mathrm{~dB}$ are considered as intermediate SNRs. As we can see on Figure 3, detection performances are still interesting for these SNRs, but they become more complex to evaluate and understand. Whereas
TABLE 2: Detection performances of the HOS (contrast), with an $\mathrm{SNR}$ of $50 \mathrm{~dB}$, in function of the size of the computation window.

\begin{tabular}{l|cc}
\hline \multirow{2}{*}{ Window size } & \multicolumn{2}{|c}{ Contrasts } \\
& Skewness & Kurtosis \\
\hline $13 \times 13$ & 77.3 & 496.2 \\
$21 \times 21$ & 209.4 & 2270.9 \\
$41 \times 41$ & 1195.4 & $2.59 \times 10^{4}$ \\
\hline
\end{tabular}

skewness values remain close to zero for SNRs below $10 \mathrm{~dB}$ for small values of $p$, negative values with high amplitude appear for SNRs greater than $-50 \mathrm{~dB}$, with a peak at $0 \mathrm{~dB}$, for high values of $p$. Therefore, for an SNR between $-50 \mathrm{~dB}$ and $10 \mathrm{~dB}$, a detection is possible by isolating pixels leading to a negative skewness. They are located close to the center of the deterministic region (see Figure 12(b)). For higher SNRs (greater than $10 \mathrm{~dB}$ ), the skewness increases regularly to reach the approximations obtained for high SNRs. For such SNRs, square structures are observed as previously described (see Figures 9(b) and 13(b)), the values decreasing more slowly near the center, but with the same structure as with high SNRs. In this case the detection method is the same as for high SNRs.

The kurtosis behaves similarly to the low SNRs case until approximately $10 \mathrm{~dB}$ with a value progressively decreasing for high values of $p$ (Figure 3(b)). The detection is possible by selecting the highest values in the kurtosis image: they are located near the center of the object (Figure 12(c)). This is similar to the low SNRs case (Figure 7(a)), but the contrast between the highest kurtosis values and the noisy background is smaller. For higher SNRs (greater than $10 \mathrm{~dB}$ ), the kurtosis increases progressively for low values of $p$, reaching the approximations derived for high SNRs. As previously described, square frames appear (Figure 13(c)), the values decreasing more slowly near the center, but with the same structure as with high SNRs. Again, the detection method is the same as for high SNRs.

The numerical values reported in Tables 3 and 4 confirm these observations, with a "low SNR"-like behavior at $0 \mathrm{~dB}$ for the kurtosis and a "high SNR"-like behavior at $20 \mathrm{~dB}$, both for the skewness and the kurtosis. However, the 


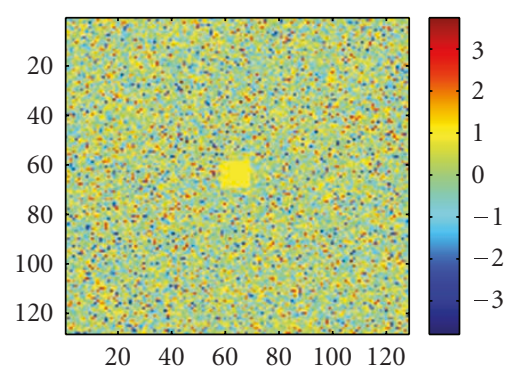

(a) Synthetic image

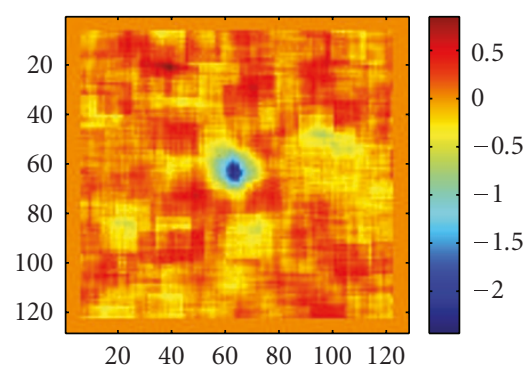

(b) Skewness

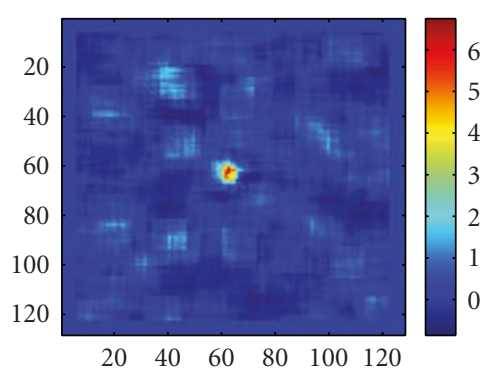

(c) Kurtosis

FIgURE 12: HOS evaluated on $13 \times 13$ windows $(\mathrm{SNR}=0 \mathrm{~dB})$.

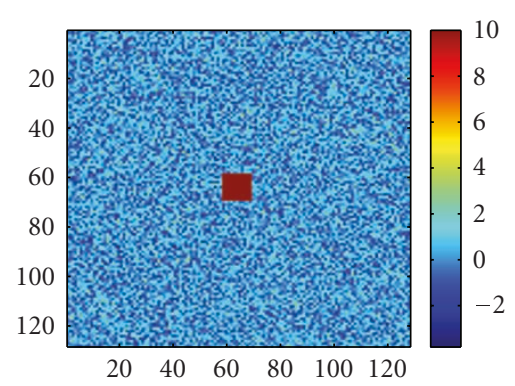

(a) Synthetic image

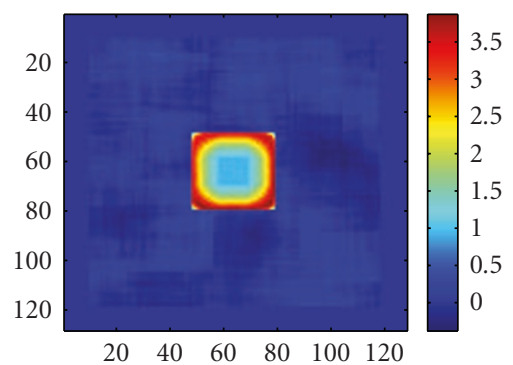

(b) Skewness

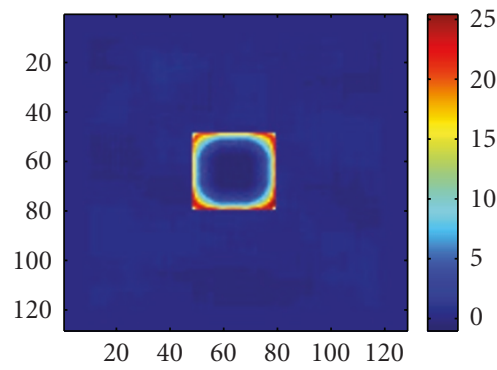

(c) Kurtosis

Figure 13: HOS evaluated on $21 \times 21$ windows $(S N R=20 \mathrm{~dB})$.

TABle 3: Detection performances of the HOS (contrast), with an $\mathrm{SNR}$ of $0 \mathrm{~dB}$, in function of the size of the computation window.

\begin{tabular}{l|cc}
\hline \multirow{2}{*}{ Window size } & \multicolumn{2}{|c}{ Contrasts } \\
& Skewness & Kurtosis \\
\hline $13 \times 13$ & 14.5 & 19.9 \\
$21 \times 21$ & 8.39 & 4.46 \\
$41 \times 41$ & 6.84 & 5.21 \\
\hline
\end{tabular}

contrasts are lower than in the previous case, but they still remain largely above the SNR of the original image.

\section{DETECTION WITH HIGH SNRs: FOCUSING OF THE RESULTS}

\subsection{Matched filtering approach}

In the case of high SNRs, square frames appear around the deterministic regions in the skewness and the kurtosis images, the highest values being in the corner of these structures. This does not allow the correct localization of the sought elements in the image. To solve this problem, a matched filtering approach is proposed in this section by performing a correlation of the HOS image with a theoretical
TABLE 4: Detection performances of the HOS (contrast), with an SNR of $20 \mathrm{~dB}$, in function of the size of the computation window.

\begin{tabular}{l|cc}
\hline \multirow{2}{*}{ Window size } & \multicolumn{2}{|c}{ Contrasts } \\
& Skewness & Kurtosis \\
\hline $13 \times 13$ & 24.4 & 75.5 \\
$21 \times 21$ & 38.8 & 131.3 \\
$41 \times 41$ & 113.3 & 384.5 \\
\hline
\end{tabular}

model of the result. The results of this focusing will only be presented on the kurtosis image, the results obtained with the skewness being similar with the corresponding theoretical model.

Knowing the size of the computation window and approximately knowing the size of the sought objects, the approximation presented in (19) is used to build a suitable model. For example, for the kurtosis image obtained on Figure 15(a), the obtained model is shown on Figure 14 (with a size of $31 \times 31$ as explained by (20)). We can see on Figure 15(b) the result obtained by the correlation of the kurtosis image with the model. In the zoomed image (Figure 15(c)), the maximum of the filtered result can be observed at the exact position of the center of the deterministic region of the original image. 


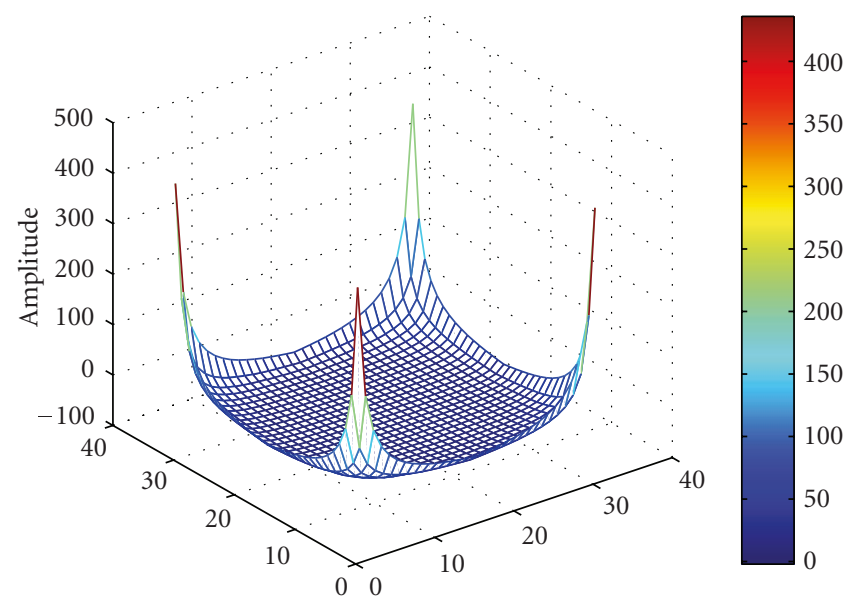

FIgURe 14: Kurtosis theoretical model used for the matched filter (window size $21 \times 21$, region size $11 \times 11$ ).

\subsection{Uncertainty regarding the size of the deterministic region}

The size of the used computation window is known (it is user-defined), but the size of the sought objects is not precisely known. As a consequence, the sizes of the structures needing to be focused in the HOS images remain partially unknown. A solution consists in taking, for the correlation, a model built with the sum of several models with different sizes. This sum is weighted in accordance with a Gaussian distribution with a mean corresponding to the most typical size, and a variance bound with the incertitude on the knowledge of this size. It is tested on an image containing two deterministic regions (Figure 11(a), described in Section 3.3). If a $25 \times 25$ model is chosen on the kurtosis image obtained with a $15 \times 15$ window (Figure 16(a)), only the wider region is correctly focused (Figure 16(b)). To solve this problem, a new model is built with a mean size of $23 \times 23$ and an uncertainty (standard deviation of the Gaussian distribution) of 3 (Figure 17). This allows a fairly good detection of the two regions (Figure 16(c)).

\subsection{Rebuilding of the region of interest: dilation with a fuzzy operator}

The focusing of the HOS results allows to have the highest values in the center of the sought region. This is very interesting for detection and localization of the region, but it does not give its shape and size. A morphological dilation is performed on the focused HOS images to solve this problem. The operator use is fuzzy [20] in order to take into account the uncertainty on the size of the sought region. The model uses the same size of uncertainty as for the matched filter. The corresponding results are presented in Section 5.2.

A simple threshold of the latter image then allows an easy detection and precise localization of the sought regions. If available, further prior knowledge about the characteristics of the object of interest can be incorporated into the model (e.g., a rectangular shape can be used instead of a square).

\section{APPLICATION IN SONAR IMAGING}

In this section, the proposed algorithm is tested on real sonar images, with application to underwater mines detection. These images are obtained by a synthetic aperture sonar (SAS), an active sonar imaging system providing high resolution images of the sea bed.

\subsection{Specificities in sonar imaging}

The sonar images used in this section represent the sea bed with different objects lying, partially or completely buried in the sea floor. When they are not buried, the objects cast a shadow on the sea bed (see, e.g., the triangular shaped shadow on Figure 19(a)). All these objects also generate some echoes (reflexion of the sound wave on the objects), these echoes being the only noticeable element in the case of buried objects. As seen on Figures 20(a) and 21(a), these objects are hardly visible and the images are seriously corrupted by a speckle noise giving a granular aspect to the image and disturbing its interpretation.

A good statistical model of this noise in the case of high resolution images is given by the Weibull law described by the following probability density function [21,22]:

$$
W_{B}(B)=\frac{\delta}{\alpha}\left(\frac{B}{\alpha}\right)^{\delta-1} \exp \left\{-\left(\frac{B}{\alpha}\right)^{\delta}\right\}, \quad B \geq 0,
$$

with $\alpha$ the scale parameter and $\delta$ the shape parameter, strictly positive.

With such a non-Gaussian distribution, background values of the skewness $S_{B}$ and the kurtosis $K_{B}$ are not null anymore (see the appendix). On real SAS data, $\delta$ is function of the resolution of the image, but it is generally approximated by 1.65 [23]. This corresponds to skewness and kurtosis values close to 1 (Figure 18).

Considering the echoes generated by the mines as deterministic elements of amplitude $A$, the SNR is defined as

$$
\rho_{W}=\frac{A}{\alpha}
$$

This assumption is simple but fair, since the echoes are generally composed of a few pixels, with values included in a limited range compared to the background (it would be difficult to model these values with a random distribution).

The approximations made in Section 3.3 remain valid in the case of high SNRs. As a matter of fact, the resulting coefficients of the highest orders ( 3 for the skewness, 4 for the kurtosis) are the same (see the terms in bold in the appendix). This induces similar detection properties with the previous case. For sonar images, the SNR as defined above is far greater than $0 \mathrm{~dB}$, even in the case of buried objects. As a conclusion, following the discussions in Section 3 on detection in the case of high or intermediate SNRs, the proposed algorithm is well suited to objects detection in SAS imaging [23]. 


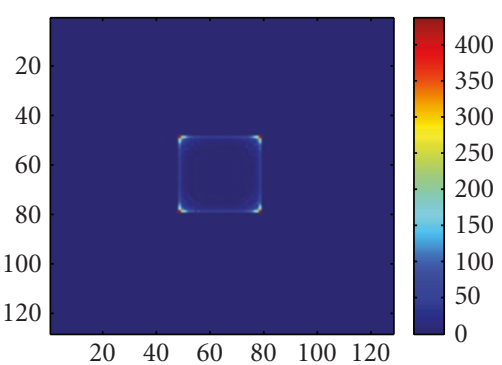

(a) Kurtosis image.

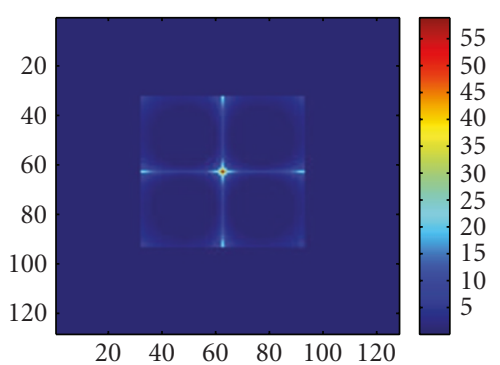

(b) Matched filtering.

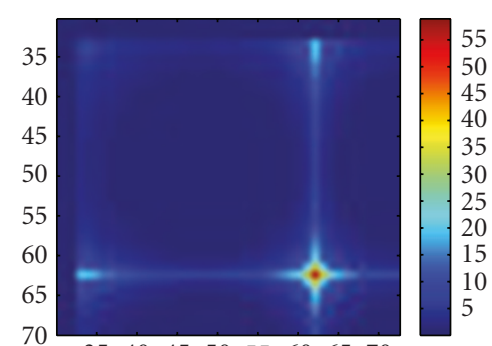

$35 \quad 40 \quad 45 \quad 50 \quad 55 \quad 6065 \quad 70$

(c) Zoom

FIGURE 15: Matched filtering on the kurtosis image (window size $21 \times 21$ ).

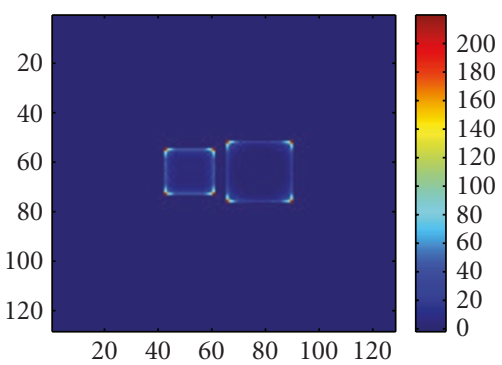

(a) Kurtosis image $(15 \times 15)$.

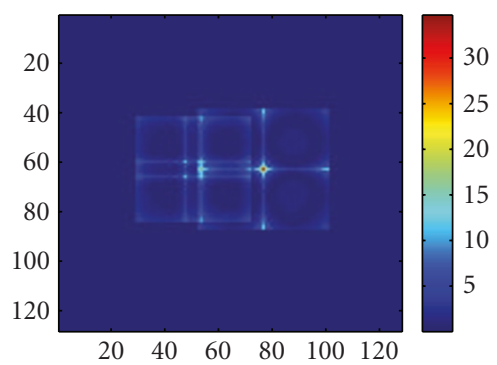

(b) Matched filtering with no uncertainty.

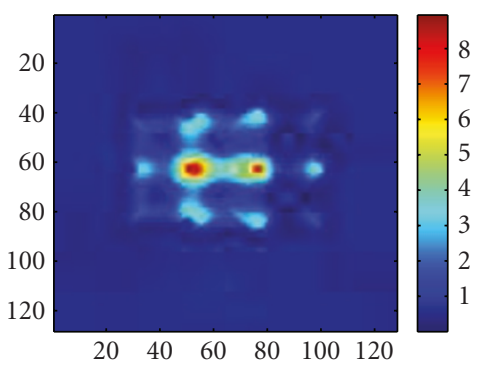

(c) Matched filtering with uncertainty.

FIGURE 16: Matched filtering on the kurtosis image of two deterministic regions with no uncertainty $(25 \times 25)$ and an uncertainty $(23 \times 23$, $\mathrm{SD}=3)$.

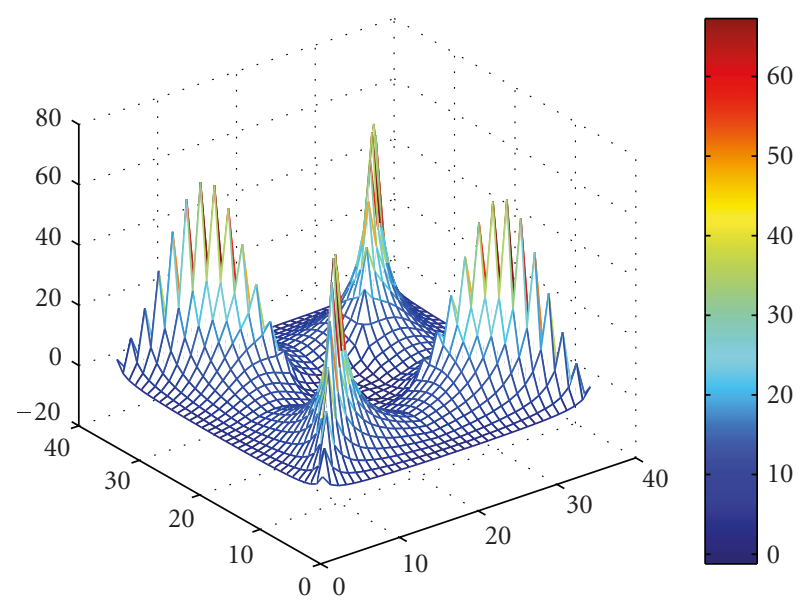

FIGURE 17: Kurtosis theoretical model used for the matched filter (mean window size $23 \times 23, \mathrm{SD}=3$ ).

\subsection{Results on SAS images}

In this section, the proposed detection method is tested on various real SAS images provided by the DGA (Délégation Générale de l'Armement, France). The resolution is defined here as the size of one pixel. This is different from the actual

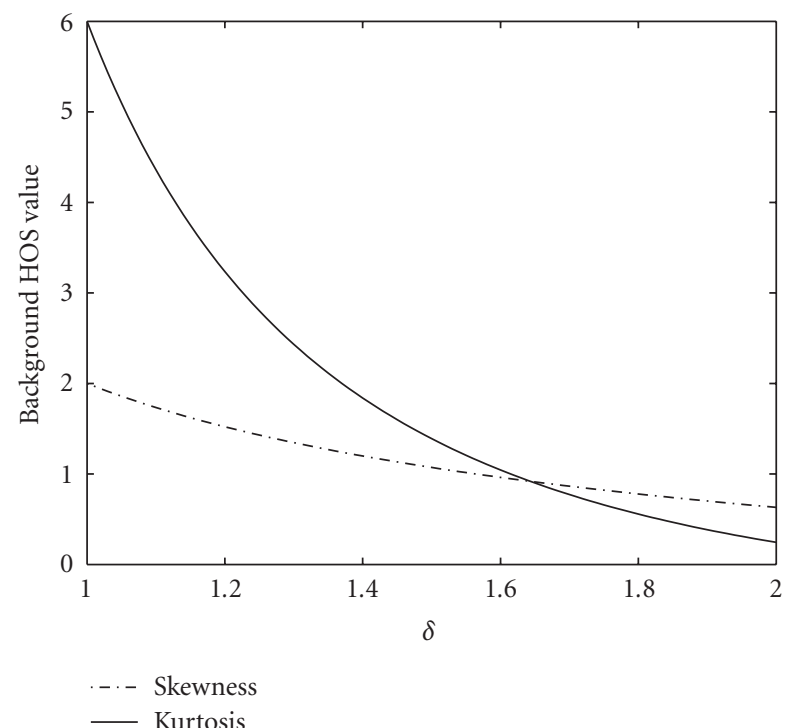

FIGURE 18: Weibull background HOS values in function of the parameter $\delta$.

system resolution, corresponding to the smallest distance between two objects that can be distinguished, which is 


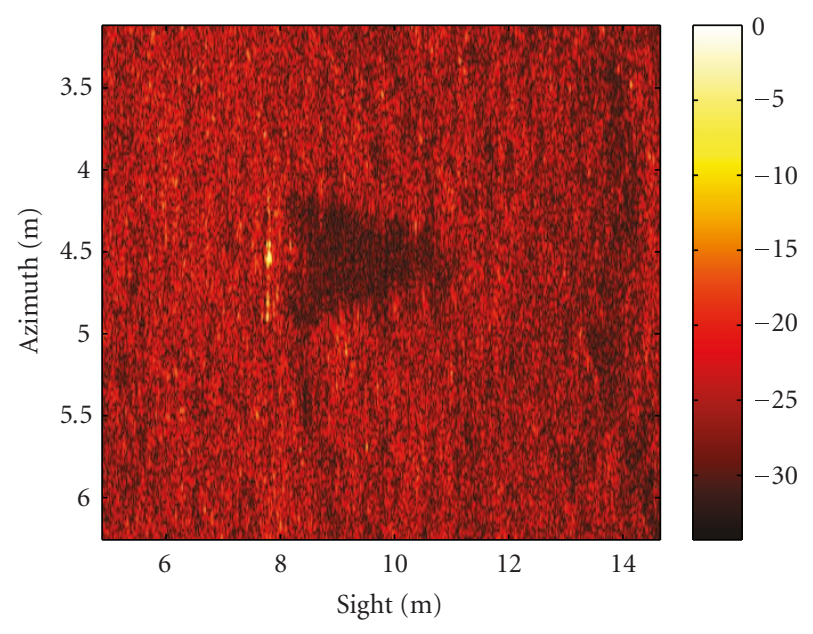

(a) SAS image

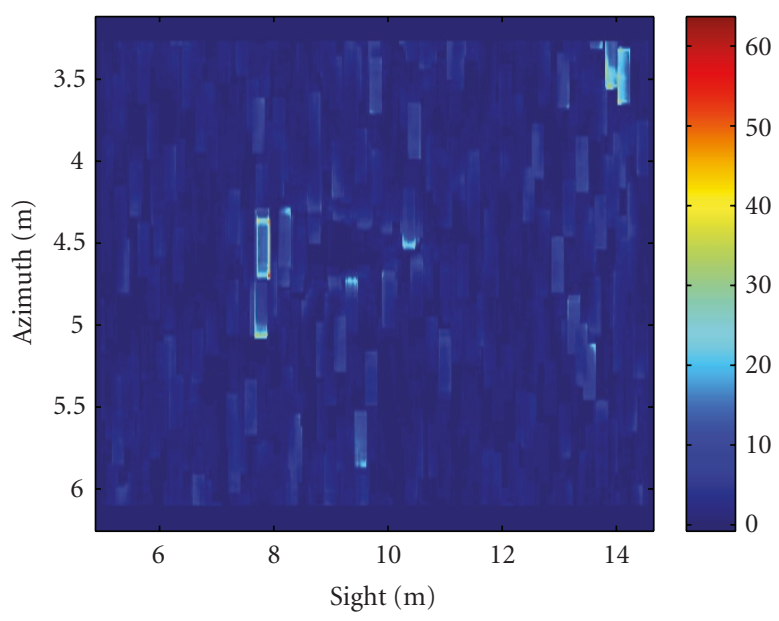

(b) Kurtosis

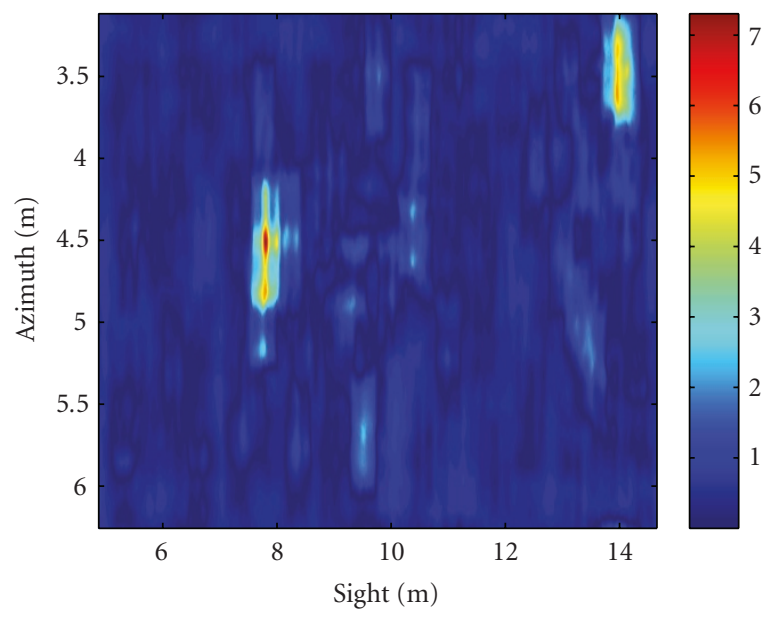

(c) Detection

FIgURE 19: Detection on the first SAS data (kurtosis $21 \times 21$, matched filtering $25 \times 25, \mathrm{SD}=3$ ). generally larger. This explains the independence of the size of the computation window and the resolution.

The first image (Figure 19(a)) contains an underwater mine lying on the sea bed. It is recognizable thanks to the shadow cast on the sea bed and the echoes generated by the object [24]. This image represents a region of $3.5 \mathrm{~m}$ by $10 \mathrm{~m}$, with a resolution of approximately $1 \mathrm{~cm}$ in both dimensions. After the computation of the kurtosis image using a $21 \times 21$ window, taking into account the dimension of the echoes and the space in between, the resulting image is matched filtered (taking into account the uncertainty on the dimension of the echoes). Figure 19(b) represents the kurtosis estimated on a sliding computation window. On the result obtained after a focusing and rebuilding process (Figure 19(c)), the two main echoes that characterize the mine are clearly detected.

The second data set is more complex: the original SAS image contains several buried or partially buried objects. This image represents a sea bed region of about $10 \mathrm{~m}$ by $10 \mathrm{~m}$, with a resolution of about $10 \mathrm{~cm}$ in both dimensions. In this image, the echoes are hardly visible apart from a partially buried cylindrical mine on the left, around sight sample 16 (Figure 20(a)). Here, the computation of the kurtosis image uses an $11 \times 11$ window (Figure 20(b)): the resolution of this image is lower than in the previous one, and the echoes thus appear as smaller objects in terms of number of pixels. The result of the matched filter is presented on Figure 20(c). This result is extremely interesting: buried objects, that were hardly visible on the original SAS image, now clearly appear. Some false alarms appearing on the lower part of the picture are due to rocks. Note that the rectangular echo on the left, created by a cylindrical mine, is well detected even though a simple square model was used for the focusing.

The third SAS image represents a region of $40 \mathrm{~m}$ by $20 \mathrm{~m}$ of the sea bed with a pixel size of about $4 \mathrm{~cm}$ in both directions $[25,26]$ (Figure 21(a)). It contains three cylindrical underwater mines: one mine is lying on the sea floor (at the top of the image), another one is partially buried (about $2 / 3$, in the middle), and the last one is completely buried under the sea floor (lower part of the picture). After the computation of the kurtosis image using a $55 \times 55$ window (Figure $21(\mathrm{~b})$ ), the result of the matched filter is presented on Figure 21(c). The result enhances the three echoes corresponding to the three mines. Note that the amplitudes in the resulting image are similar, whereas the echoes were of different amplitudes in the original SAS image. This independence is a key result of our study.

\subsection{Performance evaluation}

To quantitatively evaluate the detection performances of the proposed algorithm, ROC curves are computed. The evolution of the detection probability versus the false alarm rate is plotted when the threshold value increases. These probabilities are estimated using manually designed ground truth images. The set $A$ of pixels assumed to actually belong to the echoes is determined by an expert. The result of the algorithm for a given threshold is a set $B$ of segmented pixels (Figure 22). With $N_{A}$ the number of pixels in $A$ and $N_{A \cap B}$ the 


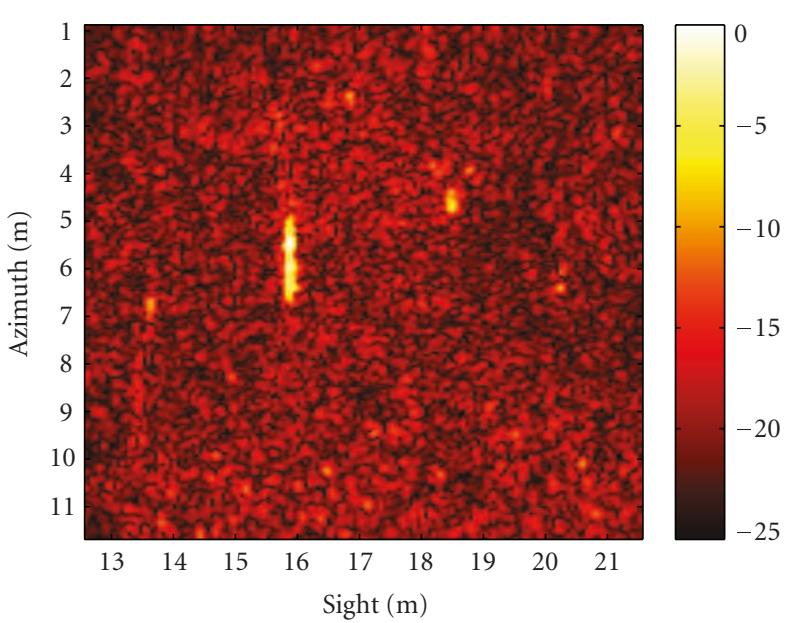

(a) SAS image

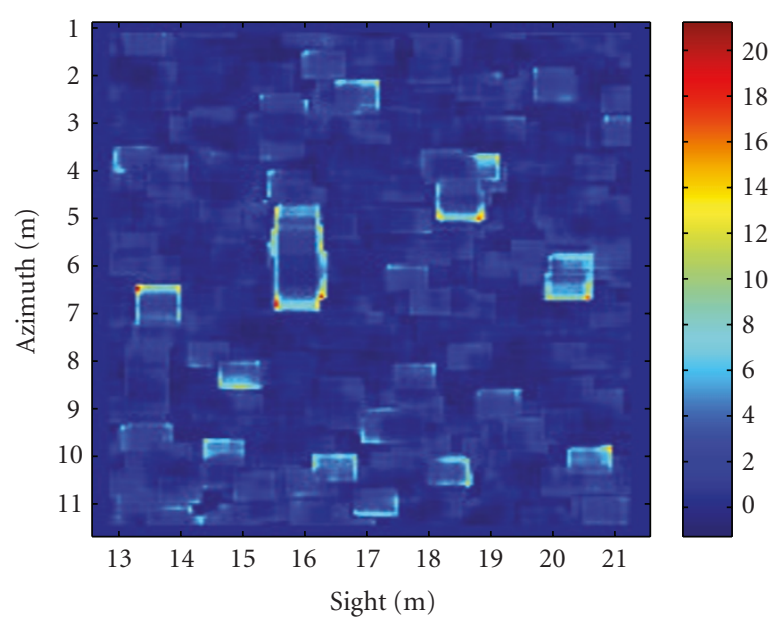

(b) Kurtosis

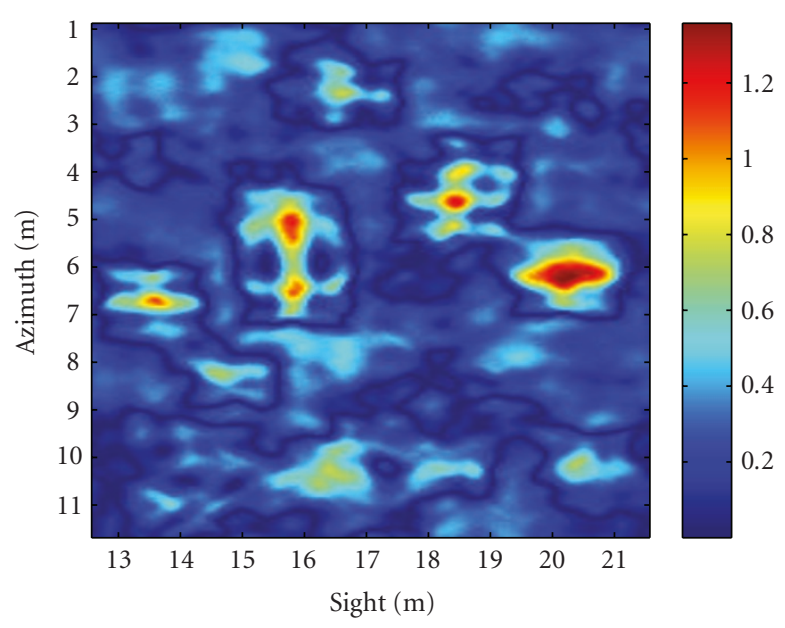

(c) Detection

Figure 20: Detection on the second SAS data (kurtosis $11 \times 11$, matched filtering $15 \times 15, \mathrm{SD}=3$ ). number of pixels in the intersection of $A$ and $B$, the detection probability $p_{d}$ is estimated as

$$
p_{d}=\frac{N_{A \cap B}}{N_{A}} .
$$

The false alarm rate $p_{f a}$ is estimated as

$$
p_{f a}=\frac{N_{\bar{A} \cap B}}{N_{\bar{A}}}
$$

with $\bar{A}$ the complement of $A\left(N_{\bar{A}}=N-N_{A}\right.$ with $N$ the size of the original image).

The proposed method using HOS is compared with the conventional detection method consisting in directly thresholding the amplitude of the original SAS data (noted "original" on the figures).

Figure 23 represents the ROC curves estimated on the results of the different process on the second (a) and the third (b) data set, respectively, where buried mines are present. From the ROC curves, both the skewness and the kurtosis clearly provide better detection performances than the conventional algorithm. The skewness seems to be more efficient than the kurtosis. As a matter of fact, the kurtosis estimator has a higher variance and thus induces a higher false alarm rate for a given detection probability.

\section{CONCLUSION}

Based on higher-order statistics, an original detection method of small deterministic regions surrounded by random noise is proposed in this paper. Two main cases are studied.

(i) In the case of low SNR, the detection can be easily performed by selecting the pixels leading to locally high values of the kurtosis. These pixels are located near the center of the sought object.

(ii) In the case of high SNRs, a matched filter is applied on the HOS images in order to obtain a precise localization of the sought elements.

A strong enhancement of the deterministic regions is obtained, thus enabling a robust detection. In the situation of intermediate SNRs, the results can be linked with the two previous cases. The robustness of the method can be emphasized, the detection being possible for high SNRs as well as for low SNRs. The results are proved to be theoretically independent from the amplitude of the sought region.

On the other hand, some prior knowledge is also required: the typical size of the sought objects and the minimal spacing between two objects should be approximately known. A hint on the SNR value (high or low?) is also required to know whether the results need to be focused or not. However, for one given application, this knowledge is usually available. Furthermore, in the focusing step, a given uncertainty can be introduced in the model to take some imprecision into account and thus increase robustness.

The proposed method is applied on real sonar (SAS) data for object detection. The use of HOS in this framework is an original contribution of this paper. Extremely promising results are obtained on various data sets, with different resolutions. This is assessed by the standard ROC curves. 


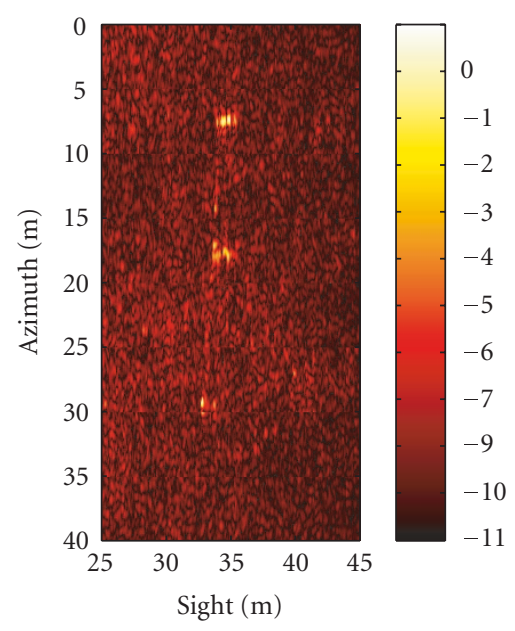

(a) SAS image

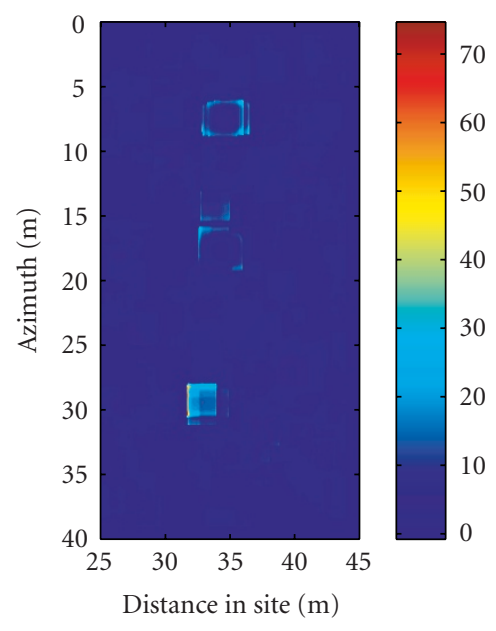

(b) Kurtosis

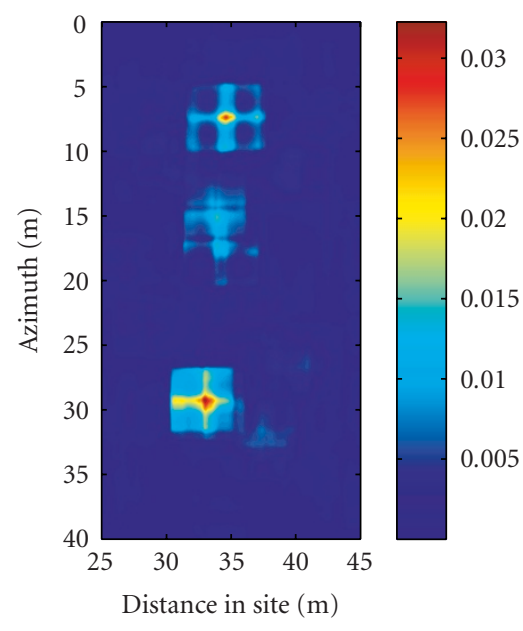

(c) Detection

FIgURE 21: Detection on the third SAS data (kurtosis $55 \times 55$, matched filtering $63 \times 63, \mathrm{SD}=3$ ).

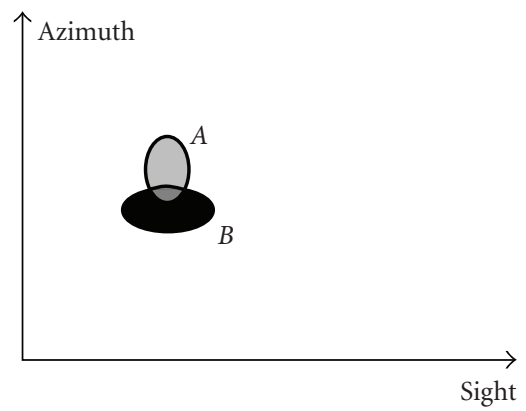

FIGURE 22: Evaluation of the detection probability and the false alarm rate: $A$ is a region considered by the expert as a "real" echo, $B$ is a region segmented at a given threshold.

Finally, one should underline the genericity and the robustness of the proposed method. As a matter of fact, the very same algorithm has been successfully applied for an application in quality control of X-ray images for a biomedical application. The corresponding results are reported in [27].

\section{APPENDIX}

\section{SKEWNESS AND KURTOSIS IN SONAR IMAGING (WEIBULL MODEL)}

We assume the noised background of our images is modeled by a Weibull law described by the following probability density function:

$$
W_{B}(B)=\frac{\delta}{\alpha}\left(\frac{B}{\alpha}\right)^{\delta-1} \exp \left\{-\left(\frac{B}{\alpha}\right)^{\delta}\right\}, \quad B \geq 0
$$

with $\alpha$ the scale parameter and $\delta$ the shape parameter, strictly positive. The $r$ th order noncentral moment $\mu_{B(r)}^{\prime}$ is given by

$$
\mu_{B(r)}^{\prime}=\alpha^{r} \Gamma\left(1+\frac{r}{\delta}\right)
$$

with $\Gamma$ the Gamma function: $\Gamma(z+1)=z !=\int_{0}^{+\infty} e^{-t} t^{z} d t$.

Noting $\gamma_{k}=\Gamma(1+k / \delta)$, we derive

$$
\begin{gathered}
S_{B}=\frac{\gamma_{3}-3 \gamma_{2} \gamma_{1}+2 \gamma_{1}^{3}}{\left(\gamma_{2}-\gamma_{1}^{2}\right)^{3 / 2}} \\
K_{B}=\frac{\gamma_{4}-4 \gamma_{3} \gamma_{1}-3 \gamma_{2}^{2}+12 \gamma_{2} \gamma_{1}^{2}-6 \gamma_{1}^{4}}{\left(\gamma_{2}-\gamma_{1}^{2}\right)^{2}} .
\end{gathered}
$$

$A$ is the amplitude of the echo. The SNR is then defined as

$$
\rho_{W}=\frac{A}{\alpha}
$$

Using the (15), we have

$$
\begin{aligned}
& \mu_{W}^{\prime}(1)=p D+(1-p) \alpha \gamma_{1}, \\
& \mu_{W}^{\prime}(2)=p D^{2}+(1-p) \alpha^{2} \gamma_{2}, \\
& \mu_{W}^{\prime}(3)=p D^{3}+(1-p) \alpha^{3} \gamma_{3}, \\
& \mu_{W}^{\prime}(4)=p D^{4}+(1-p) \alpha^{4} \gamma_{4} .
\end{aligned}
$$




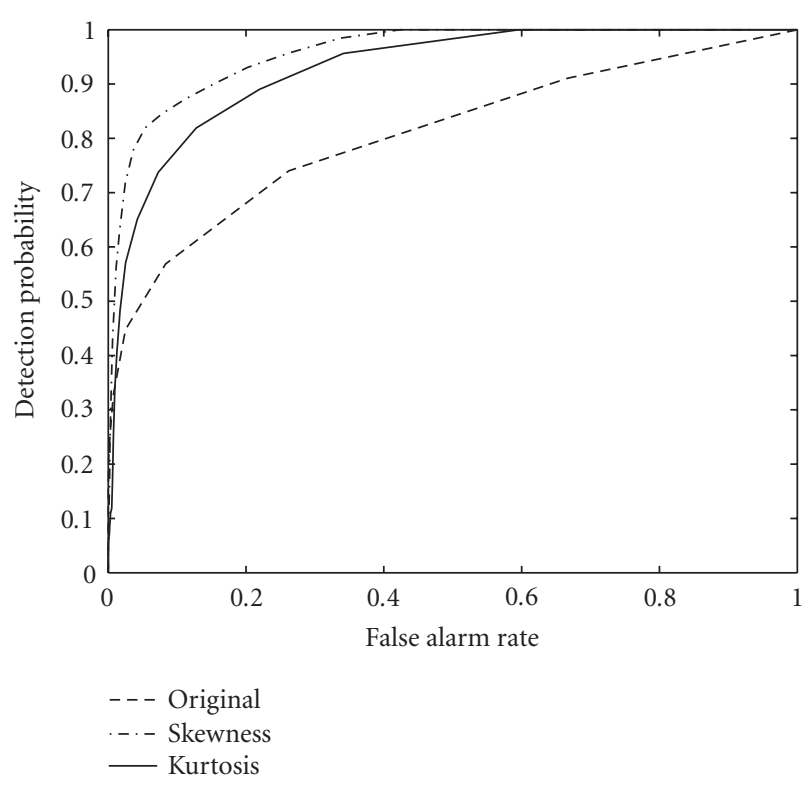

(a) Second data

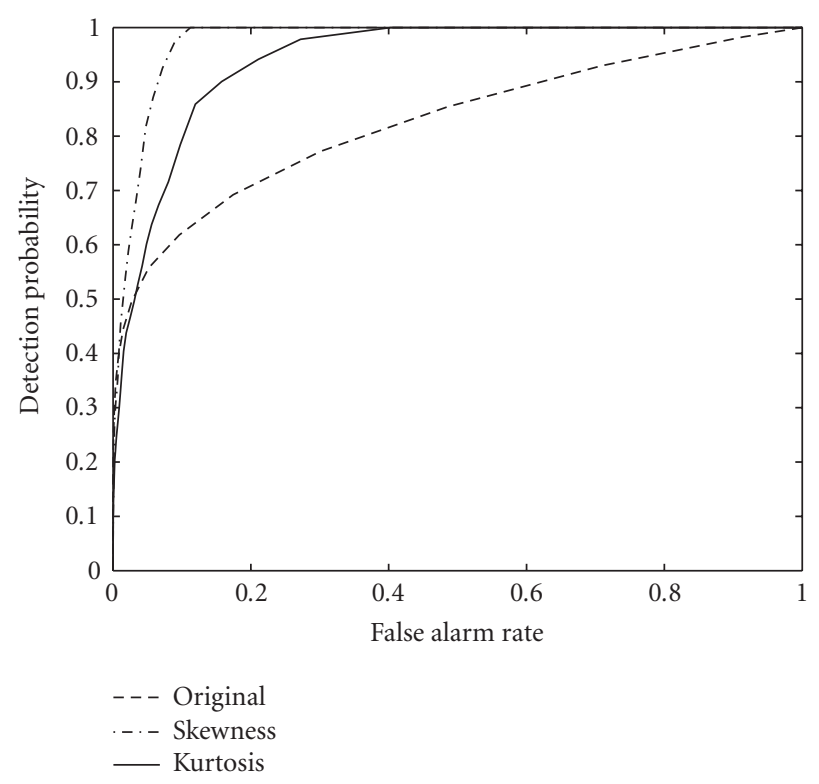

(b) Third data

FIgURE 23: Performances of the HOS on the second and third SAS data compared with an amplitude threshold.

We derive

$$
\begin{aligned}
& \mu_{W(2)}=(1-p)\left\{p A^{2}-2 \gamma_{1} p A \alpha+\left(\gamma_{2}-\gamma_{1}^{2}+\gamma_{1}^{2} p\right) \alpha^{2}\right\} \\
& \mu_{W(3)}=(1-p)\left\{p(1-2 p) A^{3}-3 p(1-2 p) \gamma_{1} A^{2} \alpha\right. \\
& -3 p\left(\gamma_{2}-2 \gamma_{1}^{2}+2 \gamma_{1}^{2} p\right) A \alpha^{2} \\
& +\cdots+\left[\gamma_{3}-3 \gamma_{2} \gamma_{1}+2 \gamma_{1}^{3}\right. \\
& \left.\left.+\gamma_{1}\left(3 \gamma_{2}-4 \gamma_{1}^{2}\right) p+2 \gamma_{1}^{3} p^{2}\right] \alpha^{3}\right\} \\
& \mu_{W(4)}=(1-p)\left\{p\left(1-6 p+6 p^{2}\right) A^{4}\right. \\
& -4 p\left(1-6 p+6 p^{2}\right) \gamma_{1} A^{3} \alpha \\
& -\cdots-6 p\left[\gamma_{2}-2 \gamma_{1}^{2}-2\left(\gamma_{2}-4 \gamma_{1}^{2}\right) p\right. \\
& \left.-6 \gamma_{1}^{2} p^{2}\right] A^{2} \alpha^{2} \\
& -\cdots-4 p\left[\gamma_{3}-6 \gamma_{2} \gamma_{1}+6 \gamma_{1}^{3}+6 \gamma_{1}\left(\gamma_{2}-2 \gamma_{1}^{2}\right) p\right. \\
& \left.+6 \gamma_{1}^{3} p^{2}\right] A \alpha^{3} \\
& +\cdots+\left[\gamma_{4}-3 \gamma_{2}^{2}-4 \gamma_{3} \gamma_{1}+12 \gamma_{2} \gamma_{1}^{2}-6 \gamma_{1}^{4}\right. \\
& +\left(3 \gamma_{2}^{2}+4 \gamma_{3} \gamma_{1}-24 \gamma_{2} \gamma_{1}^{2}+24 \gamma_{1}^{4}\right) p \\
& +\cdots+12 \gamma_{1}^{2}\left(\gamma_{2}-3 \gamma_{1}^{2}\right) p^{2} \\
& \left.\left.+24 \gamma_{1}^{4} p^{3}-6 \gamma_{1}^{4} p^{4}\right] \alpha^{4}\right\}+3 \mu_{W(2)}^{2} .
\end{aligned}
$$

We include the SNR $\rho_{W}$ :

$$
\begin{array}{r}
\frac{\mu_{W(2)}}{\alpha^{2}}=(1-p)\left\{\mathbf{p} \boldsymbol{\rho}_{\mathbf{W}}^{2}-2 \gamma_{1} p \rho_{W}+\left(\gamma_{2}-\gamma_{1}^{2}+\gamma_{1}^{2} p\right)\right\} \\
\frac{\mu_{W(3)}}{\alpha^{3}}=(1-p)\left\{\mathbf{p}(\mathbf{1}-2 \mathbf{p}) \boldsymbol{\rho}_{\mathrm{W}}^{3}-3 p(1-2 p) \gamma_{1} \rho^{2}\right. \\
-3 p\left(\gamma_{2}-2 \gamma_{1}^{2}+2 \gamma_{1}^{2} p\right) \rho \\
+\cdots+\left[\gamma_{3}-3 \gamma_{2} \gamma_{1}+2 \gamma_{1}^{3}\right. \\
\left.\left.+\gamma_{1}\left(3 \gamma_{2}-4 \gamma_{1}^{2}\right) p+2 \gamma_{1}^{3} p^{2}\right]\right\} \\
\frac{\mu_{W(4)}}{\alpha^{4}}=(1-p)\left\{\mathbf{p}\left(\mathbf{1}-\mathbf{6} \mathbf{p}+\mathbf{6} \mathbf{p}^{2}\right) \boldsymbol{\rho}_{\mathrm{W}}^{4}\right. \\
-4 p\left(1-6 p+6 p^{2}\right) \gamma_{1} \rho_{W}^{3} \\
-\cdots-6 p\left[\gamma_{2}-2 \gamma_{1}^{2}-2\left(\gamma_{2}-4 \gamma_{1}^{2}\right) p\right. \\
\left.-6 \gamma_{1}^{2} p^{2}\right] \rho^{2} \\
-\cdots-4 p\left[\gamma_{3}-6 \gamma_{2} \gamma_{1}+6 \gamma_{1}^{3}\right. \\
\left.+6 \gamma_{1}\left(\gamma_{2}-2 \gamma_{1}^{2}\right) p+6 \gamma_{1}^{3} p^{2}\right] \rho \\
+\cdots+\left[\begin{array}{c}
\gamma_{4}-3 \gamma_{2}^{2}-4 \gamma_{3} \gamma_{1}+12 \gamma_{2} \gamma_{1}^{2}-6 \gamma_{1}^{4} \\
+\left(3 \gamma_{2}^{2}+4 \gamma_{3} \gamma_{1}-24 \gamma_{2} \gamma_{1}^{2}+24 \gamma_{1}^{4}\right) p \\
+\cdots+12 \gamma_{1}^{2}\left(\gamma_{2}-3 \gamma_{1}^{2}\right) p^{2}
\end{array}\right. \\
\left.\left.+24 \gamma_{1}^{4} p^{3}-6 \gamma_{1}^{4} p^{4}\right]\right\}+\frac{3 \mu_{W(2)}^{2}}{\alpha^{4}} .
\end{array}
$$

We have then obviously

$$
\begin{gathered}
S_{W}\left(\rho_{W}, p\right)=\frac{\mu_{W(3)}}{\mu_{W(2)}^{3 / 2}}, \\
K_{W}\left(\rho_{W}, p\right)=\frac{\mu_{W(4)}}{\mu_{W(2)}^{2}}-3 .
\end{gathered}
$$




\section{ACKNOWLEDGMENTS}

The authors wish to thank the Groupe d'Etudes SousMarines de l'Atlantique (DGA/DET/GESMA, France) and TNO Defence, Security and Safety (The Netherlands) for providing SAS data in this work supported by GESMA.

\section{REFERENCES}

[1] Special issue on higher-order statistics, IEEE Transactions on Acoustics, Speech, and Signal Processing, vol. 38, no. 7.

[2] Special issue on higher-order statistics, Signal Processing vol. 36, no. 3 .

[3] Special issue on higher-order statistics, Signal Processing vol. 53, no. 2-3.

[4] G. B. Giannakis, "Cumulants: a powerful tool in signal processing," Proceedings of the IEEE, vol. 75, no. 9, pp. 1333-1334, 1987.

[5] J. M. Mendel, "Tutorial on higher-order statistics (spectra) in signal processing and system theory: theoretical results and some applications," Proceedings of the IEEE, vol. 79, no. 3, pp. 278-305, 1991.

[6] M. Krob and M. Benidir, "Blind identification of a linearquadratic mixture: application to quadratic phase coupling estimation," in Proceedings of IEEE Signal Processing Workshop on Higher-Order Statistics, pp. 351-355, South Lake Tahoe, Calif, USA, June 1993.

[7] J. R. Fonollosa and C. T. Nikias, "Wigner higher order moment spectra: definition, properties, computation and application to transient signal analysis," IEEE Transactions on Signal Processing, vol. 41, no. 1, pp. 245-266, 1993.

[8] G. Jacovitti and G. Scarano, "Hybrid nonlinear moments in array processing and spectrum analysis," IEEE Transactions on Signal Processing, vol. 42, no. 7, pp. 1708-1718, 1994.

[9] P. Comon, "Independent component analysis. A new concept?” Signal Processing, vol. 36, no. 3, pp. 287-314, 1994.

[10] D. Yellin and E. Weinstein, "Criteria for multichannel signal separation," IEEE Transactions on Signal Processing, vol. 42, no. 8, pp. 2158-2168, 1994.

[11] H.-L. N. Thi and C. Jutten, "Blind source separation for convolutive mixtures," Signal Processing, vol. 45, no. 2, pp. 209229, 1995.

[12] G. Jacovitti, "Applications of higher order statistics in image processing," in Proceedings of International Signal Processing Workshop on Higher Order Statistics, pp. 241-247, Chamrousse, France, July 1991.

[13] C. Coroyer, C. Jorand, and P. Duvaut, "ROC curves of skewness and kurtosis statistical tests: application to textures," in Proceedings of the 7th European Signal Processing Conference (EUSIPCO '94), vol. 1, pp. 450-453, Edinburgh, Scotland, UK, September 1994.

[14] C. Avilés-Cruz, R. Rangel-Kuoppa, M. Reyes-Ayala, A. Andrade-Gonzalez, and R. Escarela-Perez, "High-order statistical texture analysis-font recognition applied," Pattern Recognition Letters, vol. 26, no. 2, pp. 135-145, 2005.

[15] A. N. Rajagopalan, A. Jain, and U. B. Desai, "Data clustering using hierarchical deterministic annealing and higher order statistics," IEEE Transactions on Circuits and Systems II: Analog and Digital Signal Processing, vol. 46, no. 8, pp. 1100-1104, 1999.

[16] S. Carrato and G. Ramponi, "Edge detection using generalized higher-order statistics," in Proceedings of IEEE Signal Pro- cessing Workshop on Higher-Order Statistics, pp. 66-70, South Lake Tahoe, Calif, USA, June 1993.

[17] G. Ramponi and S. Carrato, "Performance of the Skewness-ofGaussian (SoG) edge extractor," in Proceedings of the 7th European Signal Processing Conference (EUSIPCO '94), vol. 1, pp. 454-457, Edinburgh, Scotland, UK, September 1994.

[18] D. Alexandrou, C. de Moustier, and G. Haralabus, "Evaluation and verification of bottom acoustic reverberation statistics predicted by the point scattering model," Journal of the Acoustical Society of America, vol. 91, no. 3, pp. 1403-1413, 1992.

[19] M. G. Kendall and A. Stuart, The Advanced Theory of Statistics, vol. 1, Charles Griffin, London, UK, 2nd edition, 1963.

[20] V. di Gesu, M. C. Maccarone, and M. Tripiciano, Mathematical Morphology based on Fuzzy Operators, Fuzzy Logic, edited by R. Lowen and M. Roudens, Kluwer Academic, Boston, Mass, USA, 1993.

[21] M. Mignotte, C. Collet, P. Pérez, and P. Bouthemy, “Threeclass Markovian segmentation of high-resolution sonar images," Computer Vision and Image Understanding, vol. 76, no. 3, pp. 191-204, 1999.

[22] F. Maussang, J. Chanussot, and A. Hétet, "Automated segmentation of SAS images using the mean-standard deviation plane for the detection of underwater mines," in Proceedings of MTS/IEEE Oceans Conference, vol. 4, pp. 2155-2160, San Diego, Calif, USA, September 2003.

[23] F. Maussang, J. Chanussot, and A. Hétet, "On the use of higher order statistics in SAS imagery," in Proceedings of IEEE International Conference on Acoustics, Speech and Signal Processing (ICASSP '04), vol. 5, pp. 269-272, Montreal, Quebec, Canada, May 2004.

[24] A. Hétet, "Evaluation of specific aspects of synthetic aperture sonar, by conducting at sea experiments with a rail, in the frame of mine hunting systems design," in Proceedings of the 5th European Conference on Underwater Acoustics (ECUA '00), pp. 439-444, Lyon, France, July 2000.

[25] A. Hétet, M. Amate, B. Zerr, et al., "SAS processing results for the detection of buried objects with a ship-mounted sonar," in Proceedings of the 7th European Conference on Underwater Acoustics (ECUA '04), pp. 1127-1132, Delft, The Netherlands, July 2004.

[26] J. C. Sabel, J. Groen, M. E. G. D. Colin, et al., "Experiments with a ship-mounted low frequency SAS for the detection of buried objects," in Proceedings of the 7th European Conference on Underwater Acoustics (ECUA '04), pp. 1133-1138, Delft, The Netherlands, July 2004.

[27] F. Maussang and J. Chanussot, "Utilisation des statistiques d'ordres supérieurs en contrôle qualité de détecteurs de rayons X," in Proceedings of the 20th GRETSI Symposium on Signal and Image Processing, pp. 117-120, Louvain-la-Neuve, Belgium, September 2005.

F. Maussang is graduated in electrical engineering from the Institut National Polytechnique de Grenoble (INPG), Grenoble, France, in 2002, and received the Ph.D. degree from the University of Grenoble 1, in 2005. He prepared his Ph.D. degree in Laboratoire des Images et des Signaux (LISGIPSA), Grenoble, on image processing and data fusion for detection in acoustical imaging. Since September 2006, he is teaching electronics at the University of Paris $\mathrm{X}$ and he is working in

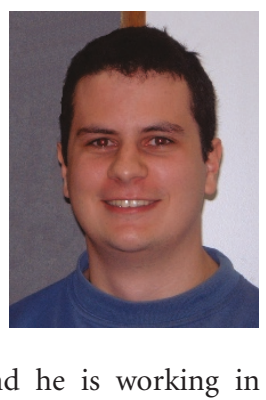


the research team GEA (Groupe d'Electromagnétisme Appliqué). His research interests are today multistatic radar imagery and ground target detection. He serves as a reviewer for IEEE Transactions on Image Processing.

J. Chanussot received the Master degree in electrical engineering from the Institut National Polytechnique de Grenoble (INPG), Grenoble, France, in 1995, and the Ph.D. degree from Savoie University, Annecy, France, in 1998. In 1999, he was with the Geography Imagery Perception Laboratory for the Délégation Générale de l'Armement (French National Defense Department). Since 1999, he has been an As-

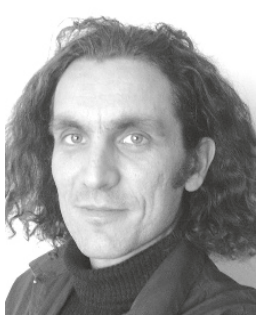
sociate Professor of signal and image processing at INPG and has been working at the Laboratoire des Images et des Signaux (LISGIPSA), Grenoble. His research interests include statistical modeling, multicomponent image processing, nonlinear filtering, remote sensing, and data fusion. He is an Associate Editor of the IEEE Geoscience and Remote Sensing Letters (2005-) and for Pattern Recognition (2006-2008). He is the Cochair of the IEEE Geoscience and Remote Sensing Society Data Fusion Technical Committee (20052007) and a Member of the Machine Learning for Signal Processing Technical Committee of the IEEE Signal Processing Society (20062008). He is a Senior Member of the IEEE (2004). He serves as a regular reviewer for various conferences (IEEE ICASSP, IEEE ICIP, and ACIVS).

A. Hétet was born in 1968 and received a B.Eng. degree in naval electrical engineering in 1988 from Ecole Technique Normale (ETN). He was graduated from the Ecole Nationale Supérieure d'Ingénieurs d'Etudes en Techniques de l'Armement (ENSIETA) in 1995 and received the Ph.D. degree from the University Pierre et Marie Curie (Paris 6 ) in acoustics and electronics in 2003 . He is currently Ingénieur des Etudes et Tech-

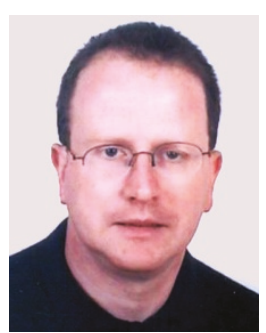
niques d'Armement (IETA) employed in the Underwater Robotics Department at the Groupe d'Etudes Sous Marines de l'Atlantique, DGA/GESMA, Brest, France, acting as an underwater robotics and mine countermeasures sonar expert. His research interests include all aspects of underwater robotics sensors and vision, advanced imagery sonar systems, automatic target detection and recognition, marine environment characterization, synthetic aperture sonar, proud and buried object detection and classification. He is currently an Associated Member in the laboratory for Extraction and Exploitation of Information in Uncertain Environments $\left(\mathrm{E}^{3} \mathrm{I}^{2}\right.$ EA3876) at ENSIETA.

M. Amate is graduated from the Ecole Nationale Supérieure des Ingénieurs des Etudes et Techniques d'Armement (ENSIETA), Brest, France, and received the M.S. degree in digital signal and image processing from Cranfield University, United Kingdom. She is currently working as underwater mine warfare expert in the Groupe d'Etude Sous-Marines de l'Atlantique (DGA/DET/GESMA), Brest, France, since 2003. Her interests include synthetic aperture sonar (SAS) systems. 